\title{
Exploring Conformational Landscapes using Trap and Release Tandem Ion Mobility Spectrometry
}

Aurélien Le Fèvre, ${ }^{\dagger}$ Philippe Dugourd, ${ }^{\star}$ and Fabien Chirot $^{* \dagger}$

†Univ Lyon, Université Claude Bernard Lyon 1, CNRS, UMR5280 Institut des Sciences Analytiques, F69100, 5 rue de la Doua, VILLEURBANNE, France

†niv Lyon, Université Claude Bernard Lyon 1, CNRS, UMR5306 Institut Lumière Matière, F-69100, 5 rue de la Doua, VILLEURBANNE, France.

*email : fabien.chirot@univ-lyon1.fr

ABSTRACT: The dynamics and thermodynamics of structural changes in isolated glu-fibrinopeptide B (GluFib) were investigated by tandem ion mobility spectrometry (IMS). Doubly protonated GluFib ${ }^{2+}$ ions were first selected by IMS and then stored for a controlled duration in a thermalized ion trap. Temperature-induced conformational changes were finally monitored by IMS as a function of trapping time. Based on this procedure, isomerization rates and equilibrium populations of the different conformers were determined as a function of temperature. We demonstrate that the measured thermodynamic quantities can be directly compared to simulated observables from ensemble molecular modeling, based on appropriate order parameters. We obtained good qualitative agreement with replica-exchange molecular dynamics simulations based on the AMOEBA force field and processed using the weighted histogram analysis method. This suggests that the balance between Coulomb repulsion and optimal charge solvation is the main source of the observed conformational bi-stability. Our results emphasize the differences between the kinetically-driven quasi-equilibrium distributions obtained after collisional activation and the thermodynamically-driven distributions from the present equilibrium experiments, due to entropic effects. As a consequence, our measurements do not only allow straightforward determination of Arrhenius activation energies, but also yields the relative enthalpy and entropy changes associated to a structural transition.

Structural flexibility is an essential feature in the response of complex molecular systems to external stimuli. Numerous bimolecular processes involve conformational changes in biomolecules, especially in proteins. Moreover, the function of synthetic molecular machines often relies on structural changes. The flexibility of a molecule depends on the features of its energy landscape, whose valleys and saddle points define the paths and the kinetics of conformational changes. Probing energy landscapes nevertheless remains an experimental challenge. In particular, due to the generally high dimensionality of the conformational space, structure-related observables are often indirect, and only partially describe the conformational state of the system under study. Moreover, flexible systems often co-exist under different forms, the contribution of which may be difficult to disentangle from an ensemble measurement. In this context, gas-phase approaches are appealing because they allow isolating and studying separately different species co-existing as a mixture in the condensed phase.

Among gas-phase structural probes, ion mobility spectrometry (IMS), in combination with mass spectrometry (MS) has recently emerged as one of the most powerful and versatile techniques. ${ }^{1-}$ ${ }^{5}$ IMS can be used to separate isomers of molecular systems, and to gain insight in their structure, through comparison with molecular modeling. ${ }^{6-9}$ IMS structural resolution nevertheless remains relatively modest, however it can be combined with other complementary gas phase techniques, including different flavors of spectroscopic measurements, ${ }^{10-14}$ as well as frag- 
mentation. ${ }^{15}$ Beyond simple separation, IMS-MS data can provide insight into molecular flexibility, through the interpretation of the observed collision cross section (CCS) distributions as a projection of the accessible conformational space. Its exploitation in the context of "nativeMS" largely builds on this idea. Alternatively, conformational changes triggered by collisional, ${ }^{16,17}$ or photon excitation, ${ }^{18,19}$ or even by temperature changes, ${ }^{20-22}$ can be directly monitored by IMS. This allows a direct exploration of conformational transition pathways for isolated molecules. Such approaches are particularly powerful when applied within an IMS/IMS scheme, as pioneered by the group of D. Clemmer. ${ }^{23}$ In an IMS/IMS experiment, specific isomeric species can be isolated as a function of their arrival time after a first IMS separation. Then, energy can be transferred to the selected species by collisional activation or laser irradiation. Finally, a second IMS separation allows for the characterization of conformational changes, from a well-defined initial state. Following this scheme, Pierson et al. obtained a precise description of the conformational landscape of triply-protonated Bradykinin, using collisional activation to trigger structural changes. ${ }^{24}$ Based on a calibration of the collision energy using fragmentation rate constants measurements, they were able to provide values for the energy barriers between the main states, and to infer their relative energies. The approach is not limited to ground-state processes: the group of $E$. Bieske, ${ }^{18,25,26}$ and our group ${ }^{19,27}$ reported direct photo-isomerization measurements by adding a laser excitation step between the two IMS stages.

In the following, we explore an alternative approach to the exploration of isomerization pathways, based on kinetic measurements in an IMS/IMS scheme. Kinetic measurements using IMS have already been reported, especially to follow the time-evolution of the composition of a mixture in solution as a function of temperature. ${ }^{28,29}$ This kind of measurement can yield thermodynamic quantities, such as enthalpy and entropy differences between the different states, or the height of the energy barriers connecting them. Temperature-dependent experiments also enable direct characterization of the "melting" of protein assemblies by IMS. ${ }^{30,31} \mathrm{Be}-$ sides, slow structural reorganizations in biomolecular ions after desolvation could be emphasized by trapping them for variable durations before IMS measurements. ${ }^{32}$ Moreover, the impact of gas phase isomerization kinetics on the shape of IMS arrival time distributions (ATDs) has long been exploited to determine the associated kinetics and thermodynamic constants. This is possible if the timescales involved are on the order of that of the measurements. ${ }^{33-35}$ Our approach consists in trapping IMS-selected ions for a controlled amount of time, at a controlled temperature. A second IMS separation is then performed, followed by a MS measurement. We used this approach to characterize the relaxation of a peptide from a state defined by the selection, to an equilibrium. These results were then interpreted in terms of thermodynamics quantities defining the local conformational landscape of the peptide. Finally, we discuss the interest of the present approach compared to collisional activation.

\section{EXPERIMENTAL SECTION}

Sample. Lyophilized [Glu1]-Fibrinopeptide B (GluFib) was purchased from Sigma-Aldrich (sequence: Glu-Gly-Val-Asn-Asp-Asn-Glu-Glu-GlyPhe-Phe-Ser-Ala-Arg). Solutions were prepared in a mixture of water and acetonitrile (1:1) to a concentration of $25 \mu \mathrm{mol} . \mathrm{L}^{-1}$. To promote protonation for positive electrospray ionization, we added $0.1 \%$ trifluoroacetic acid. The solution was sprayed directly.

Tandem Ion Mobility. We used a homebuilt tandem-ion mobility instrument previously described in ref. 36. A scheme of the instrument is provided in Scheme S1, and details on the experimental setup are provided as Supplementary Information. IMS/IMS is made possible through the association of two drift tubes in series. Briefly, ions can be selected using an ion gate at the end of the first drift tube (DT1). Then, they are 
either subjected to collisional activation, or stored in an ion trap (Trap 2 on Scheme S1) for a controlled duration, at a controlled temperature. After a second IMS separation in DT2, ions are finally detected as a function of their mass-tocharge ratio. Under these conditions, if the drift time of the ions trough DT2 differs from that selected after DT1, it implies that their mobility have changed between the two IMS stages. This can be attributed either to a change in their mass-to-charge ratio, visible in the mass spectrum, or to isomerization.

Molecular Modeling. Replica-exchange molecular dynamics simulations (REMD) ${ }^{37}$ were performed to explore the conformational landscape of the investigated peptide and produce ensembles of structures and observables comparable to our experimental data. ${ }^{7}$ We used a custom python implementation of REMD based on the Python plugin of the OpenMM library. ${ }^{38}$ Simulations were performed in vacuo with the AMOEBA polarizable force field, ${ }^{39}$ using 16 replicas propagated at temperatures ranging from 300 to $800 \mathrm{~K}$. Using the procedure described in the Supplementary Information section, we obtained a pool of 30000 structures per replica.

For comparison with the experiment, CCS were calculated for selected structures using the trajectory method, ${ }^{40}$ as implemented in the Collidoscope software. ${ }^{41}$ Since CCS calculations are relatively expensive, we used the gyration radius as an order parameter to characterize the free energy surface (FES) of the peptide from the statistics on sample structures at the different temperatures, based on the weighted histograms analysis method (WHAM). ${ }^{8,42}$ Structural observables were extracted from the sample structures using the MDAnalysis toolkit. ${ }^{43,44}$

\section{Derivation of rate constants and thermodynam-}

ic quantities. Our aim is to extract thermodynamics observables from the experimental observable: the time evolution of the populations as a function of temperature. Populations were determined by fitting the experimental ATDs with two Gaussian functions (see Figure S2), yielding intensities $I_{A}^{\text {exp }}$ and $I_{B}^{\text {exp }}$, which were normalized so that $I_{A}^{\text {exp }}+I_{B}^{\text {exp }}=1$ at any time.

To extract rate constants from this data, we used a fitting procedure based on the analytical expression of the time evolution of the populations obtained from a simple two-state model (see Supplementary Information for details). The fits of the experimental data yield $k_{A B}$ and $k_{B A}$, the rate constants for isomerization from state $A$ to state $B$ and from state $B$ to state $A$, respectively. The corresponding Arrhenius activation energies $\mathrm{E}_{A B}^{*}$ and $\mathrm{E}_{B A}^{*}$ can finally be extracted from the evolution of these rate constants as a function of temperature.

For long enough timescales $(t \gg 1 / k)$, the populations reach a steady state, which we considered as an equilibrium. Consequently, the quantity $K_{A B}=I_{B}(\infty) / I_{A}(\infty)=k_{A B} k_{B A}$ correspond to an equilibrium constant. We estimated the enthalpy and entropy of isomerization, $\Delta \mathrm{H}_{\mathrm{AB}}$ and $\Delta \mathrm{S}_{\mathrm{AB}}$, from the evolution of this quantity with temperature.

\section{RESULTS AND DISCUSSION}

Two populations are separated by ion mobility. The arrival time distribution (ATD) recorded for $[\text { GluFib }+2 \mathrm{H}]^{2+}$ ions ( $\left.\mathrm{m} / \mathrm{z} 786.28\right)$ is plotted in Figure S1. Two peaks are visible in the distribution. We further denote $A$ and $B$ the populations corresponding to the peak centered at $20.8 \mathrm{~ms}$, and $21.6 \mathrm{~ms}$, respectively. While the width of peak $A$ is attributable to diffusion broadening, ${ }^{45}$ peak $B$ is slightly broader (see Figure S2). This may indicate that peak $B$ is a mixture of unresolved conformers. In the following, the intensities reported for populations $A$ and $B$ were all determined using a fixed width for the Gaussian functions used in the fit ( 0.39 and 0.58 ms FWHM for peak $A$ and $B$, respetively).

Since all ions considered for this ATD have the same charge state, the conformation of population $A$ is expected to be more compact than that of population $B$. In terms of CCS, we measured $312 \AA^{2}$, and $324 \AA^{2}$ for $A$ and $B$, respectively. 
These values are very close, as expected from the arrival times.

(a)
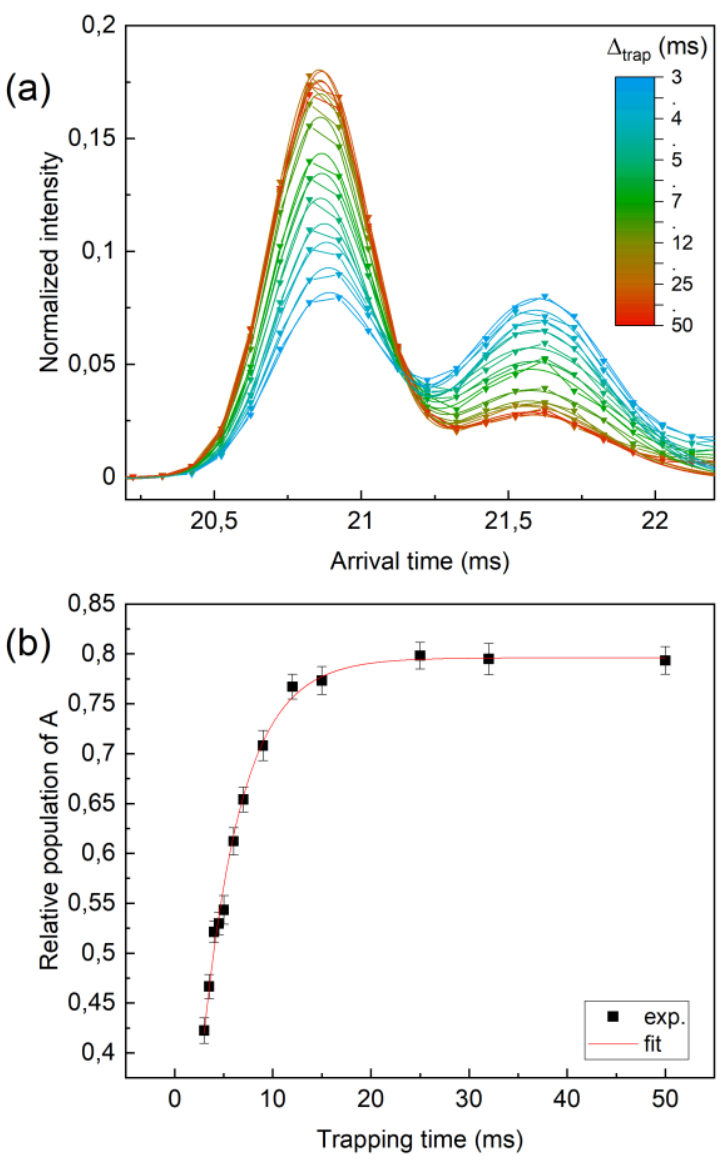

Figure 1: (a) Arrival time distributions for doubly-protonated GluFib $^{2+}$ ions recorded after selection of population B. In each case ions are stored for a controlled duration $\Delta_{\text {trap }}$ in Trap 2 at $\mathrm{T}=$ $338 \mathrm{~K}$. The different curves correspond to different storage times. (b) Evolution of the relative proportion of population A as a function of trapping time. The solid line correspond to a fit of the data using equation $\mathrm{S} 2$.

ATDs obtained in IMS-IMS mode, with minimal residence time in Trap 2 are also displayed in Figure S1 (in this case the delay $\Delta_{\text {trap }}$ between selection and injection in DT2 is set to $5 \mathrm{~ms}$, which roughly corresponds to the drift time of the ions between the selection gate and the gate of Trap 2). When the selection window is set to the arrival time of population A, the ATD consists in a single peak centered at $20.8 \mathrm{~ms}$, as expected if no isomerization occurs after selection. Likewise, the ATD recorded after selection of population $B$ is peaked at $21.6 \mathrm{~ms}$, the arrival time of B. However, it also displays a marked shoulder in the region assigned to $A$. This shoulder is not attributed to IMS selection (populations with closer drift times could be isolated using the present setup ${ }^{19}$ ), but rather to spontaneous $B \rightarrow A$ isomerization, occurring after selection. The evolution of the populations with increasing trapping time supports this interpretation (see below).

Temperature-dependent kinetic measurements. After IMS-selections, ions were trapped for a controlled duration, at different temperatures, before recording their ATDs in the second drift region. Figure 1.(a) displays ATDs recorded for ions selected in the $B$ state and trapped at $338 \mathrm{~K}$ before analysis. The different ATDs correspond to different storage times in the trap. Yet for the shortest times, peak $A$ is dominant, indicating that efficient $B \rightarrow A$ isomerization has occurred after selection. As the storage time increases, population B decreases further until a steady state is reached, after about 20-30 ms. This behavior is better illustrated by the plot of the time evolution of the relative population of state $A$ on Figure 1.(b). The functional of equation S2 nicely reproduces the observed trend, which allows determining the associated rate constants from the fitting parameters: $k_{A B}^{338 K}=$ $0.051 \pm 0.003 \mathrm{~ms}^{-1}$, and $k_{B A}^{338 K}=0.199 \pm$ $0.004 \mathrm{~ms}^{-1}$ (the reported errors refer to the standard deviations from the fit). From the longtime behavior, we can also determine the associated equilibrium constant $K_{A B}^{338 K}=0.26 \pm$ 0.02 . The same procedure was applied for different trapping temperatures, ranging from $295 \mathrm{~K}$ to $411 \mathrm{~K}$. The corresponding data are provided as supplementary material (Figure S3). For the temperatures above $356 \mathrm{~K}$, the kinetics were too fast for the transient population evolution to be visible; then only the equilibrium values were determined.

Derivation of thermodynamic quantities. The evolution of the rate constants and the equilibrium constant as the function of the inverse temperature are represented in Figure 2.(a) and (b), respectively. Linear fits were performed on each dataset. From the slope of the Arrhenius plots on Figure 2.(a), we determined the Arrhenius activation energies: $E_{A B}^{*}=65 \pm 7 \mathrm{~kJ}^{*} \mathrm{~mol}^{-1}$, and 
$\mathrm{E}_{B A}^{*}=54 \pm 17 \mathrm{~kJ} \mathrm{~mol}^{-1}$. On the other hand, the relative enthalpy of states $B$ and $A$ can be obtained from the slope of the Van't Hoff plot in Figure 2.(b), yielding $\Delta H_{A B}=15.5 \pm$ $0.6 \mathrm{~kJ} . \mathrm{mol}^{-1}$. This positive value is in agreement with the observation that spontaneous isomerization occurs from $B$ to $A$, yet at room temperature, indicating that $A$ is more stable than $B$. It also worth noting that the value of $\Delta H_{A B}$ is compatible with the difference between the above activation energies: $\quad \mathrm{E}_{A B}^{*}-\mathrm{E}_{B A}^{*}=11 \pm$ $5 \mathrm{~kJ} \cdot \mathrm{mol}^{-1}$.


Figure 2: (a) Arrhenius plots of the measured isomerization rate constants as a function of the inverse temperature in both $A \rightarrow B$ (red) and $\mathrm{B} \rightarrow \mathrm{A}$ (black) directions, with the associated linear fits. (b) Van't Hoff plot of the measured equilibrium constants as a function of the inverse temperature, with a linear fit. The shaded areas correspond to the $95 \%$ confidence bands of the fits.

Figure 2.(b) can be further exploited since the intercept of the fit provides an estimate of the entropy difference between the two states: $\Delta S_{A B}=33 \pm 2 \mathrm{~J} \cdot \mathrm{mol}^{-1} \cdot \mathrm{K}^{-1}$. This implies that $\mathrm{B}$ is entropically favored, and may then become dominant at high temperatures.

Similarly, the relative entropy of the transition state between $A$ and $B$ be could in principle be inferred from the results in Figure 2.(a), for example using Eyring's equation for the isomerization rate constants. ${ }^{46}$ However, the uncertainties on the intercepts of the linear fits were too large for meaningful values to be derived. All experimentally-derived thermodynamic quantities are listed in Table 1.

Bi-stability is qualitatively reproduced by molecular modeling. In order to gain structural insight in populations $A$ and $B$, we used the ensembles generated from REMD simulations. Since systematic CCS calculations on thousands of structures are relatively costly, the gyration radius $R g$ was used as a rough descriptor of the compactness of the structures. Indeed, the CCS is in general fairly well correlated with $R g^{2}{ }^{8}$ The gyration radii distributions of the structures sampled during the parallel MD runs are bimodal in the temperature range corresponding to the experiments, as illustrated in Figures S4. In all distributions below $600 \mathrm{~K}$, the dominant peak lies at about $\operatorname{Rg}^{2}=40 \AA^{2}$, and a second minor peak is centered at $\operatorname{Rg}^{2}=45 \AA^{2}$. Structures with higher gyration radii and thus higher CCS are also visible, and become more populated as temperature increases.

As a first picture of the sampled energy landscape, Figure 3.(a) displays the inherent structures determined from optimization of 5000 instantaneous configurations sampled along the $425 \mathrm{~K}$ replica. We used a hierarchical clustering algorithm (fcluster algorithm from scipy ${ }^{47}$ ) to identify structural similarities between the inherent structures. As a distance criterion for clustering, we used the root-mean-square deviation between corresponding backbone atoms in different structures. A dendrogram resulting 


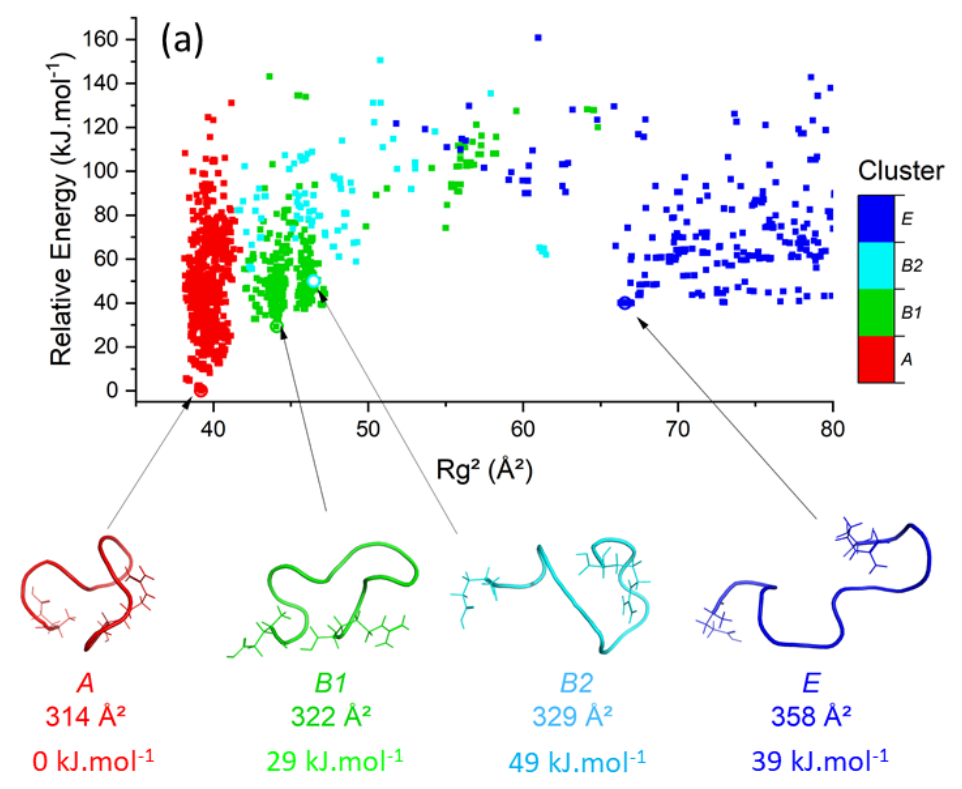

(b)

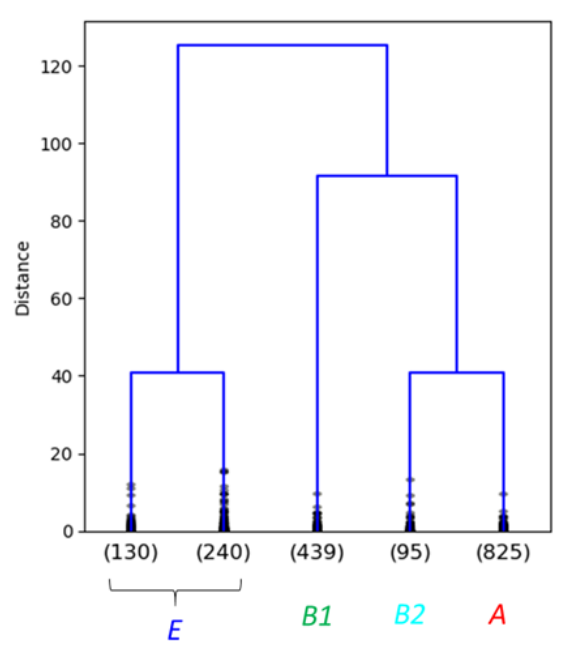

Figure 3: (a) Relative energies of the inherent structures from 5000 instantaneous snapshots of a MD run at $425 \mathrm{~K}$ as a function of their gyration radius. The colors correspond to the different clusters determined by a hierarchical clustering algorithm, based on interatomic distances. The lowest lying structure in each cluster is sketched below. The corresponding calculated CCS are provided, as well as the energies relative to the global minimum. (b) Dendrogram resulting from the hierarchical clustering procedure, truncated to display the last five clusters. Two clusters, corresponding to the most extended structures were grouped under the name $E$. The numbers in parenthesis correspond to the number of inherent structures in each cluster.

and the inherent structures in Figure 3.(a) were colored according to the cluster to which they belong. Most of the structures in the same cluster fall in a well-defined region of the conformational space defined by $\mathrm{Rg}^{2}$ vs the energy. The clusters were tentatively denoted $A, B 1, B 2$ and $E$, based on the average gyration radii of the corresponding structures.

It appears from the dendrogram in Figure 3.(b), that structures in cluster $E$ are the most structurally distinct from the others. This can be understood considering that these structures form an ensemble of highly unfolded conformations, with relatively large structural dispersity, in line with the relatively broad $\mathrm{Rg}^{2}$ range covered by this cluster. In contrast, the most compact structures, grouped in cluster $A$, all display very close gyration radii, as expected from a conformational family with a well-defined structuration. Additionally, this cluster correspond to the most populated state during the simulation, at the considered temperature. It also contains the lowest energy structure with a CCS matching that measured for population A. We therefore identify this cluster to the population $A$ isolated in the experiment. Based on similar arguments, both clusters $B 1$ and $B 2$ could contribute to the observed population B, as far as CCS is concerned. Indeed, the relatively broader $\mathrm{Rg}^{2}$ range occupied by those clusters, is consistent with the observation that peak $B$ in the ATDs was broader than peak $A$. It also gives further support to the above hypothesis that population B can access a broader range of configurations than population $A$ and may thus be entropically favored. Interestingly, structures from cluster $B 2$, although globally higher in energy than those from $B 1$, are more structurally similar to the structures from cluster $A$. Namely, as illustrated by the superimposition of the backbone of the lowest laying isomers from each cluster (Figure S5), structures from the $B 2$ and $A$ clusters share similar arrangement of their backbone on the $\mathrm{C}$-terminal 
side. The relatively higher $\mathrm{Rg}^{2}$ values displayed by $B 2$ structures mainly originate from a relative unfolding of their N-terminal moiety. In contrast, Figure S5.(b) shows that structures from the B1 cluster display a completely different arrangement, with their $\mathrm{N}$-terminus and $\mathrm{C}$-terminus moieties oriented in an opposite direction as compared to $A$ structures. Transition from $A$ to $B 1$ would then obviously require partial unfolding of the terminal regions. In this context, $B 2$ structures might represent intermediates in the transition between the more stable, and more compact $A$ and $B 1$ families.

\begin{tabular}{|c|c|c|c|}
\hline \multicolumn{2}{|c|}{ Experiment } & \multicolumn{2}{c|}{ Simulation } \\
\hline$\Delta \boldsymbol{H}_{\boldsymbol{A B}}$ & $15.5 \pm 0.6$ & $\Delta H_{A B}$ & 18 \\
\hline $\boldsymbol{\Delta S}_{\boldsymbol{A B}}$ & $33 \pm 2$ & $\Delta S_{A B}$ & 27 \\
\hline $\boldsymbol{E}_{\boldsymbol{A B}}^{*}$ & $65 \pm 7$ & $\Delta H_{A B}^{\#}$ & 23 \\
\hline & & $\Delta S_{A B}^{\#}$ & 36 \\
\hline $\boldsymbol{E}_{\boldsymbol{B A}}^{*}$ & $54 \pm 17$ & $\Delta H_{B A}^{\#}$ & 5 \\
\hline & & $\Delta S_{B A}^{\#}$ & 8 \\
\hline
\end{tabular}

Table 1: Thermodynamic quantities derived from the experimental data and from the simulated free energy surfaces. Enthalpies are in $\mathrm{kJ} \cdot \mathrm{mol}^{-1}$ and entropies in $\mathrm{J} \cdot \mathrm{mol}^{-1} \cdot \mathrm{K}^{-1}$.

Reconstructed free energy surface and qualitative interpretation of the observed bi-stability. Based on the good agreement between our simulated distributions and the experimental data, we used WHAM to estimate 1-dimensonal free energy surfaces, taking the gyration radius as an order parameter. Such surfaces computed for different temperatures in the range $360 \mathrm{~K}$ to 500 $\mathrm{K}$ are represented in Figure S6.(a). Two wells separated by a barrier are visible, in good qualitative agreement with the experimental observations. The deepest well, centered on $\mathrm{Rg}^{2}=41$ $\AA^{2}$, was identified to the conformational state $A$ considered in the two-state model. The shallowest well at $47 \AA^{2}$ was thus identified to state B.

The relative energy between the minima of clusters $A$ and $B 1$, which we denote $A O$ and $B O$, respectively (Figure S7), provides an estimate of the enthalpy difference between the corresponding wells. It is on the same order of magnitude than the measured $\Delta H_{A B}$, although somewhat higher. However, the present simulation does not yield any direct counterpart to the other experimental observables (activation energies and relative entropy of the wells). In order to push the quantitative comparison with the experiment further, we adopted a practical approach by determining the relative Gibbs free energy between each well $\Delta G_{A B}(T)$, and between each well and the top of the barrier $\left(\Delta \mathrm{G}_{\mathrm{AB}}^{\#}(T)\right.$ and $\left.\Delta \mathrm{G}_{\mathrm{BA}}^{\#}(T)\right)$ directly from the WHAM FESs at different temperatures. The linear dependence of these free energies with temperature (Figure S6.(b)) allows to estimate the associated enthalpies and entropies from their intercept and slopes, respectively. The corresponding values are listed in Table 1 , together with their experimental counterparts.

The relative enthalpy $\Delta H_{A B}$ and entropy $\Delta S_{A B}$ of the two states are fairly well reproduced in this case. In contrast, although Arrhenius activation energies should be very close to the relative enthalpies of the transition state, the simulated $\Delta H^{\#}$ are clearly much lower than their experimental counterpart. Several reasons may be invoked to explain this discrepancy. Firstly, the simulated values were estimated from a onedimensional projection of the free energy surfaces, using the gyration radius as a proxy for the experimental observable, which forces caution in the comparison. Secondly, the force field might inaccurately reproduce part of the energetics, which would be critical regarding the small energy differences between the considered structures.

Based on the qualitative match between experimental and simulated data, we investigated the origin of the observed conformational bistability. One important point on which the simulation agrees with the experiment is the relatively small energy difference between $A$ and $B$, on the order of the typical binding energy of a single $\mathrm{H}$-bond in a peptide. ${ }^{48}$ Beyond $\mathrm{H}$-bonds however, the folding of a peptide in the absence of solvent is mainly ruled by electrostatic (charge-charge, and charge-dipole) interactions. ${ }^{49-51}$ Since two positive charges are present in the investigated peptide sequence, 
Coulomb repulsion has to be a key factor in the stability of the different structures. The charged moieties are nevertheless closer in the lowest energy structure $A O$ than in the most stable configuration identified in cluster $\mathrm{B}, B O$ ( $9 \AA$ in $A O$ vs $10.2 \AA$ in $B 0$ ). It is not uncommon that the most stable structure for multiply charged flexible systems, like peptides or synthetic polymers, is not the one where the charges are further apart. Self-solvation of the charges by polar groups is often more favorable, which promotes globular structures where the flexible chain is wrapped around the charges. ${ }^{52,53}$

The role of the above stabilization or destabilization factors in the case of doubly-protonated GluFib was evaluated through the analysis of the present pool of inherent structures. The number of $\mathrm{H}$-bonds in each structure was estimated from the atomic coordinates, considering the relative positions (angle and distance) of the potential donor and acceptor groups in the peptide. The separation between the charged moieties was also systematically estimated via the distance between the $\mathrm{N}$-terminal nitrogen atom and the $\mathrm{C}_{\zeta}$ atom in the side chain of Arg14. Finally, as an evaluation of the degree of solvation of the charged groups by polar moieties (especially carbonyl groups), we determined the number of oxygen atoms within $2.8 \AA$ of each hydrogen in the terminal amino group, and in the guanidinium group of Arg14.

Interestingly, the number of $\mathrm{H}$-bonds does not vary dramatically from one cluster to the other (Figure S8.(a)). In contrast, the distance between charged moieties increases as the structures get less compact (Figure S8.(b)). Conversely, charge solvation, as estimated by the number of contacts in Figure S8.(c), is significantly more efficient in cluster $A$ than in cluster $B 1$. Moreover, as visible in Figure 57 on the structure of $A O$, the relative configuration of the side chains of Arg14 and Phe11 is compatible with additional charge solvation through $\pi$-charge interactions.

Based on the above, we tentatively suggest that the observed bi-stability results from the inter- play between the de-stabilizing Coulomb repulsion and the optimal self-solvation of the charges, which appears as the key factor in the present case. Furthermore, the hypothesis of cluster $B 2$ representing an intermediate between clusters $A$ and $B 1$, is also consistent with the associated distributions in Figure S8.(b) and Figure S8.(c), which lay in between those for clusters $A$ and $B 1$.

Comparison to collisional activation and interpretation of the quasi-equilibrium distribution. A widely spread method to explore the conformational landscape of flexible molecular systems consists in monitoring their isomerization pathways through collisional activation experiments. Simple activation before IMS analysis merely provides qualitative information on the underlying conformational landscape, potentially exploitable to derive identification patterns. ${ }^{17}$ However, careful analysis of isomerization threshold from IMS/IMS experiments is needed to estimate activation energies. ${ }^{24}$

We performed collisional activation experiments after selecting each of the identified populations $A$ and $B$. The ATDs after activation of IMS selected species are displayed in Figure 4. Isomerization can be monitored starting from both populations. If the activation voltage is increased after selection of $A$, population $B$ increases. Conversely, an increase of population $A$ is observed after collisional activation of $B$ species. For highenough voltages, the populations reach the same stationary state, independent of the initial population. In this stationary regime, A represents $18 \%$ of the total population. Interestingly, the evolution of the populations is slightly nonmonotonic when B is initially selected (see inset in Figure 4).

The high voltage behavior corresponds to what was denoted by Pierson et al. a quasiequilibrium ( $Q E)$ regime. ${ }^{54}$ In this regime, the increase of the internal energy of the system due to collisional activation is high enough to allow multiple interconversion events between the accessible states. After activation, the cooling 
caused by low energy collisions with the buffer gas is often fast enough to prevent further isomerization. As a consequence, the populations trapped in each state reflects the relative isomerization rates in the $\mathrm{QE}$ regime. In the present two-state situation, a larger population in state $B$ means that, under collisional activation, the net isomerization rate from $A$ to $B$ is higher than that in the opposite direction.

Interpreting this observation in terms of the relative stability between the two states in not trivial since the higher population in state $B$ may be explained either based on energetic or entropic arguments. On the one hand, collisional activation can act as an annealing stage, allowing relaxation to more energetically stable structures. For example, peptides can be trapped in metastable conformations after electrospray ionization and desolvation. In this case, collisional activation can trigger relaxation to structures more stable in the gas phase. ${ }^{24}$ This explanation can be discarded in the present case because IMS/IMS experiments gave evidence for spontaneous isomerization from state $B$ to state $A$, without collisional activation. This implies that state $A$ is more stable than state $B$. Consequently, the predominance of $B$ in the QE distribution has to be attributed to an entropic stabilization at the relatively high temperatures reached during collisional activation.

This interpretation also accounts for the nonmonotonic evolution of the populations at lower collisional activation, when the selected population is B (inset in Figure 4). The relative proportion of $A$ first increases for activation voltages below $40 \mathrm{~V}$, and decreases for higher voltages before it reaches a steady state at about $50 \mathrm{~V}$. The increase at low voltages is the signature that the ions have reached an effective temperature at which the isomerization rate from $B$ to $A$ is high enough for significant isomerization to occur during activation (i.e. within typically $10 \mu \mathrm{s}$ using $40 \mathrm{~V}$ activation). If $\mathrm{A}$ lies at relatively lower energy than $B$, the activation energy for the reverse process (isomerization from $A$ to $B$ ) is slightly higher. Thus, the isomerization rate is lower at the same effective temperature. Therefore, the collision voltage threshold for $A$ to $B$ isomerization to be observable is expected to be slightly higher than that for B to A - this is indeed consistent with Figure 4 . We thus interpret the presence of a maximum in the red curve in Figure 4 as the signature of an intermediate regime, where isomerization is allowed only from B to $A$.

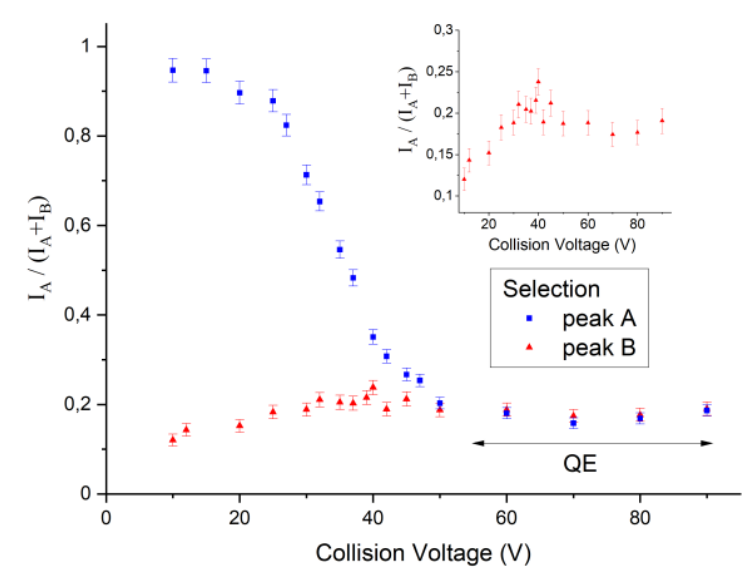

Figure 4: Evolution of the relative intensity of population of A as a function of the activation voltage when the population selected before activation is A (blue) or B (red). The existence of a stationary distribution in the quasi-equilibrium (QE) regime is emphasized. The inset is a zoom on the maximum of the red curve.

An entropic stabilization of $B$ is consistent with the sign of the above-derived $\Delta S_{A B}$. Based on the measured values of $\Delta S_{A B}$ and $\Delta H_{A B}$, state $\mathrm{B}$ would become predominant at temperatures above $457 \mathrm{~K}$. Effective temperatures on the order of $500 \mathrm{~K}$ were indeed reported for ions undergoing relatively harsh drift conditions in travelling wave, ${ }^{55}$ or trapped IMS devices. ${ }^{56-59}$ Although GluFib displays much more degrees of freedom than the "thermometer ions" used in the above studies, it is likely that the high fields applied in the activation zone are sufficient to reach such internal temperatures.

The present results emphasize important pitfalls in the interpretation of collisional activation experiments. Firstly, the attribution of the predominance of state B to entropic stabilization would not have been possible without IMS/IMS measurements showing that state $B$ is metastable. Entropic effects are indeed difficult to tackle 
using activation measurements alone, as already emphasized in ref. 24. Secondly, our data illustrate that the IMS distributions measured after collisional activation may not always faithfully represent the actual QE distribution. Namely in the present case, spontaneous $B \rightarrow A$ isomerization necessarily occurs after activation, within the timescale of the measurement. Such effect may also lead to erroneous estimation of isomerization thresholds.

\section{CONCLUSIONS}

By measuring temperature-dependent isomerization rate constants and equilibrium distributions, we were able to derive a detailed picture of the local conformational landscape of a peptide. Namely, enthalpy and entropy differences between the different states involved were determined. We showed that these observables can be further exploited with the help of molecular modeling in order to gain insight in the mechanisms at work. In the present case, the observed conformational bistability could be qualitatively reproduced using molecular mechanics simulations, indicating that the competition between Coulomb repulsion and optimal solvation of the charged groups are the main driving forces. Because thermalization is required, the proposed approach is not as straightforward to implement as collisional activation experiments. However, we showed the interest of temperature-dependent measurements, in complement to collisional activation, to disentangle enthalpic and entropic contributions in isomerization processes, and to take kinetics effects into account. Finally, the proposed methodology consists in a gas-phase transposition of a basic thermochemistry experiment. It is thus potentially applicable to any kind of isomerresolved gas-phase reaction, i.e. not limited to isomerization.

\section{ACKNOWLEDGEMENT}

This work was funded by ANR (grant $n^{\circ} 17-C E 29-$ 0013-01). The authors thank Dr. Florent Calvo for critical reading of the manuscript, and for providing his WHAM code.

\section{REFERENCES}

(1) McCabe, J. W.; Hebert, M. J.; Shirzadeh, M.; Mallis, C. S.; Denton, J. K.; Walker, T. E.; Russell, D. H. The IMS Paradox: A Perspective on Structural Ion Mobility-Mass Spectrometry. Mass Spectrom. Rev. 2020, 10.1002/mas.21642.

(2) Kalenius, E.; Groessl, M.; Rissanen, K. Ion Mobility-Mass Spectrometry of Supramolecular Complexes and Assemblies. Nat. Rev. Chem. 2019 $3(1), 4-14$.

(3) Stuchfield, D.; Barran, P. Unique Insights to Intrinsically Disordered Proteins Provided by Ion Mobility Mass Spectrometry. Curr. Opin. Chem. Biol. 2018, 42, 177-185.

(4) Eyers, C. E.; Vonderach, M.; Ferries, S.; Jeacock, K.; Eyers, P. A. Understanding Protein-Drug Interactions Using Ion Mobility-Mass Spectrometry. Curr. Opin. Chem. Biol. 2018, 42, 167-176.

(5) Gabelica, V.; Marklund, E. Fundamentals of Ion Mobility Spectrometry. Curr. Opin. Chem. Biol. 2018, 42, 51-59.

(6) Marklund, E. G.; Degiacomi, M. T.; Robinson, C. V.; Baldwin, A. J.; Benesch, J. L. P. Collision Cross Sections for Structural Proteomics. Structure 2015, 23 (4), 791-799.

(7) Chirot, F.; Calvo, F.; Albrieux, F.; Lemoine, J.; Tsybin, Y. O.; Dugourd, P. Statistical Analysis of Ion Mobility Spectrometry. I. Unbiased and Guided Replica-Exchange Molecular Dynamics. J. Am. Soc. Mass Spectrom. 2012, 23 (2), 386-396.

(8) Calvo, F.; Chirot, F.; Albrieux, F.; Lemoine, J. J.; Tsybin, Y. O.; Pernot, P.; Dugourd, P. Statistical Analysis of Ion Mobility Spectrometry. II. Adaptively Biased Methods and Shape Correlations. J. Am. Soc. Mass Spectrom. 2012, 23 (7), 1279-1288.

(9) Fernandez-Lima, F. A.; Wei, H.; Gao, Y. Q.; Russell, D. H. On the Structure Elucidation Using Ion Mobility Spectrometry and Molecular Dynamics. $J$. Phys. Chem. A 2009, 113 (29), 8221-8234.

(10) Lee, S.; Zucker, S. M.; Webber, N.; Valentine, S. J.; Clemmer, D. E.; Reilly, J. P. An Ion Mobility-Linear Ion Trap Hybrid Mass Spectrometer for Analysis of Biological Molecules; Denver, 2011.

(11) Daly, S.; MacAleese, L.; Dugourd, P.; Chirot, F. Combining Structural Probes in the Gas Phase - Ion Mobility-Resolved Action-FRET. J. Am. Soc. Mass Spectrom. 2018, 29 (1), 133-139.

(12) Lepere, V.; Le Barbu-Debus, K.; Clavaguéra, C.; Scuderi, D.; Piani, G.; Simon, A.-L.; Chirot, F.; MacAleese, L.; Dugourd, P.; Zehnacker, A. ChiralityDependent Structuration of Protonated or Sodiated Polyphenylalanines: IRMPD and Ion Mobility Studies. Phys. Chem. Chem. Phys. 2016, 18 (3), 1807-1817.

(13) Le, T. N.; Poully, J. C.; Lecomte, F.; Nieuwjaer, N.; Manil, B.; Desfrançois, C.; Chirot, F.; Lemoine, J.; Dugourd, P.; van der Rest, G.; Grégoire, G. Gas-Phase Structure of Amyloid- $\beta$ (12 - 28) Peptide Investigated by Infrared Spectroscopy, Electron Capture Dissociation and Ion Mobility Mass Spectrometry. $J$. Am. Soc. Mass Spectrom. 2013, 24 (12), 1937-1949.

(14) Seo, J.; Hoffmann, W.; Warnke, S.; Huang, X.; Gewinner, S.; Schöllkopf, W.; Bowers, M. T.; von Helden, G.; Pagel, K. An Infrared Spectroscopy Approach to Follow $\beta$-Sheet Formation in Peptide Amyloid Assemblies. Nat. Chem. 2017, 9 (1), 39-44.

(15) Song, Y.; Nelp, M. T.; Bandarian, V.; Wysocki, V. H. Refining the Structural Model of a Heterohexameric Protein Complex: Surface Induced Dissociation and Ion Mobility Provide Key Connectivity and Topology Information. ACS Cent. Sci. 2015, 1 (9), 477-487.

(16) Dilger, J.; Musbat, L.; Sheves, M.; Bochenkova, A. V.; Clemmer, D. E.; Toker, Y. Direct Measurement of the Isomerization Barrier of the Isolated Retinal Chromophore. Angew. Chemie - Int. Ed. 2015, 54 (16), 4748-4752.

(17) Tian, Y.; Han, L.; Buckner, A. C; Ruotolo, B. T. Collision Induced Unfolding of Intact Antibodies: Rapid Characterization of Disulfide Bonding Patterns, Glycosylation, and Structures. Anal. Chem. 2015, 87 (22), 11509-11515.

(18) Markworth, P. B.; Adamson, B. D.; Coughlan, N. J. A.; Goerigk, L.; Bieske, E. J. Photoisomerization Action Spectroscopy: Flicking the Protonated Merocyanine-Spiropyran Switch in the Gas Phase. Phys. Chem. Chem. Phys. 2015, 17 (39), 25676-25688.

(19) Choi, C. M.; MacAleese, L.; Dugourd, P.; Choi, M. C.; Chirot, F. Photo-Induced Linkage Isomerization in the Gas Phase Probed by Tandem Ion Mobility and Laser Spectroscopy. Phys. Chem. Chem. Phys. 2018, 20 (17), 1222312228 .

(20) El-Baba, T. J.; Woodall, D. W.; Raab, S. A.; Fuller, D. R.; Laganowsky, A.; Russell, D. H.; Clemmer, D. E. Melting Proteins: Evidence for Multiple Stable Structures upon Thermal Denaturation of Native Ubiquitin from Ion Mobility Spectrometry-Mass Spectrometry Measurements. J. Am. Chem. Soc. 2017, 139 (18), 6306-6309.

(21) Gidden, J.; Bowers, M. T. Gas-Phase Conformational and Energetic Properties of Deprotonated Dinucleotides. Eur. Phys. J. D 2002, 20 (3), 409-419.

(22) Dugourd, P.; Hudgins, R. R.; Jarrold, M. F. High-Resolution Ion Mobility Studies of Sodium Chloride Nanocrystals. Chem. Phys. Lett. 1997, 267 (12), 186-192.

(23) Korenige, S. L.; Merenbloom, S. I.; Valentine, S. J.; Jarrold, M. F. Udseth, H. R.; Smith, R. D.; Clemmer, D. E. An IMS - IMS Analogue of MS - MS. Anal. Chem. 2006, 78 (12), 4161-4174.

(24) Pierson, N. A.; Clemmer, D. E. An IMS-IMS Threshold Method for Semi-Quantitative Determination of Activation Barriers: Interconversion of Proline Cis $\leftrightarrow$ Trans Forms in Triply Protonated Bradykinin. Int. J. Mass Spectrom. 2015, $377,646-654$.

(25) Adamson, B. D.; Coughlan, N. J. A.; Markworth, P. B.; Continetti, R. E.; Bieske, E. J. An Ion Mobility Mass Spectrometer for Investigating 
Photoisomerization and Photodissociation of Molecular Ions. Rev. Sci. Instrum 2014, 85 (12), 123109

(26) Coughlan, N. J. A.; Adamson, B. D.; Gamon, L.; Catani, K.; Bieske, E. J. Retinal Shows Its True Colours: Photoisomerization Action Spectra of MobilitySelected Isomers of the Retinal Protonated Schiff Base. Phys. Chem. Chem. Phys. 2015, 17 (35), 22623-22631.

(27) Czerwinska, I.; Kulesza, A.; Choi, C.; Chirot, F.; Simon, A. L.; Far, J.; Kune, C.; De Pauw, E.; Dugourd, P. Supramolecular Influence on Cis-Tran Isomerization Probed by Ion Mobility Spectrometry. Phys. Chem. Chem. Phys. 2016, 18 (47), 32331-32336.

(28) Brown, C. J.; Woodall, D. W.; El-Baba, T. J.; Clemmer, D. E Characterizing Thermal Transitions of IgG with Mass Spectrometry. J. Am. Soc Mass Spectrom. 2019, 30 (11), 2438-2445.

(29) Poyer, S.; Choi, C. M.; Deo, C.; Bogliotti, N.; Xie, J.; Dugourd, P.; Chirot, F.; Salpin, J.-Y. Kinetic Study of Azobenzene E / Z Isomerization Using Ion Mobility-Mass Spectrometry and Liquid Chromatography-UV Detection. Analyst 2020, 145 (11), 4012-4020.

(30) Woodall, D. W.; Brown, C. J.; Raab, S. A.; El-Baba, T. J.; Laganowsky, A.; Russell, D. H.; Clemmer, D. E. Melting of Hemoglobin in Native Solutions as Measured by IMS-MS. Anal. Chem. 2020, 92 (4), 3440-3446.

(31) Woodall, D. W.; El-Baba, T. J.; Fuller, D. R.; Liu, W.; Brown, C. J.; Laganowsky, A.; Russell, D. H. Clemmer, D. E. Variable-Temperature ESI-IMSMS Analysis of Myohemerythrin Reveals Ligand Losses, Unfolding, and a NonNative Disulfide Bond. Anal. Chem. 2019, 91 (10), 6808-6814.

(32) Henderson, S. C.; Valentine, S. J.; Counterman, A. E.; Clemmer, D. E. ESI/Ion Trap/Ion Mobility/Time-of-Flight Mass Spectrometry for Rapid and Sensitive Analysis of Biomolecular Mixtures. Anal. Chem. 1999, 71 (2), 291-301. (33) Wyttenbach, T.; Pierson, N. A.; Clemmer, D. E.; Bowers, M. T. Ion Mobility Analysis of Molecular Dynamics. Annu. Rev. Phys. Chem. 2014, 65 (1), 175-196.

(34) Poyer, S.; Comby-Zerbino, C.; Choi, C. M.; MacAleese, L.; Deo, C.; Bogliotti, N.; Xie, J.; Salpin, J.-Y.; Dugourd, P.; Chirot, F. Conformational Dynamics In Ion Mobility Data. Anal. Chem. 2017, 89 (7), 4230-4237.

(35) Allen, S. J.; Eaton, R. M.; Bush, M. F. Structural Dynamics of NativeLike Ions in the Gas Phase: Results from Tandem Ion Mobility of Cytochrome $C$. Anal. Chem. 2017, 89 (14), 7527-7534.

(36) Simon, A.-L.; Chirot, F.; Choi, C. M.; Clavier, C.; Barbaire, M.; Maurelli, J.; Dagany, X.; MacAleese, L.; Dugourd, P. Tandem Ion Mobility Spectrometry Coupled to Laser Excitation. Rev. Sci. Instrum. 2015, 86 (9), 094101.

(37) Sugita, Y.; Okamoto, Y. Replica-Exchange Molecular Dynamics Method for Protein Folding. Chem. Phys. Lett. 1999, 314 (1-2), 141-151.

(38) Eastman, P.; Swails, J.; Chodera, J. D.; McGibbon, R. T.; Zhao, Y.; Beauchamp, K. A.; Wang, L.-P.; Simmonett, A. C.; Harrigan, M. P.; Stern, C. D.; Wiewiora, R. P.; Brooks, B. R.; Pande, V. S. OpenMM 7: Rapid Development of High Performance Algorithms for Molecular Dynamics. PLOS Comput. Biol. 2017 13 (7), e1005659.

(39) Shi, Y.; Xia, Z.; Zhang, J.; Best, R.; Wu, C.; Ponder, J. W.; Ren, P. Polarizable Atomic Multipole-Based AMOEBA Force Field for Proteins. J. Chem. Theory Comput. 2013, 9 (9), 4046-4063.

(40) Mesleh, M. F.; Hunter, J. M.; Shvartsburg, A. A.; Schatz, G. C.; Jarrold, M. F. Structural Information from Ion Mobility Measurements: Effects of the LongRange Potential. J. Phys. Chem. 1996, 100 (96), 16082-16086.

(41) Ewing, S. A.; Donor, M. T.; Wilson, J. W.; Prell, J. S. Collidoscope: An Improved Tool for Computing Collisional Cross-Sections with the Trajectory Method. J. Am. Soc. Mass Spectrom. 2017, 28 (4), 587-596.

(42) Kumar, S.; Rosenberg, J. M.; Bouzida, D.; Swendsen, R. H.; Kollman, P. A. THE Weighted Histogram Analysis Method for Free-Energy Calculations on Biomolecules. I. The Method. J. Comput. Chem. 1992, 13 (8), 1011-1021. (43) Gowers, R.; Linke, M.; Barnoud, J.; Reddy, T.; Melo, M.; Seyler, S.; Domański, J.; Dotson, D.; Buchoux, S.; Kenney, I.; Beckstein, O. MDAnalysis: A Python Package for the Rapid Analysis of Molecular Dynamics Simulations; 2016; pp 98-105.

(44) Michaud-Agrawal, N.; Denning, E. J.; Woolf, T. B.; Beckstein, O. MDAnalysis: A Toolkit for the Analysis of Molecular Dynamics Simulations. $J$ Comput. Chem. 2011, 32 (10), 2319-2327.

(45) Revercomb, H. E.; Mason, E. A. Theory of Plasma Chromatography, Gazeous Electrophoresis - Review. Anal. Chem. 1975, 47 (7), 970-983.

(46) Eyring, H. The Activated Complex in Chemical Reactions. J. Chem. Phys. 1935, 3 (2), 107-115

(47) Virtanen, P.; Gommers, R.; Oliphant, T. E.; Haberland, M.; Reddy, T.; Cournapeau, D.; Burovski, E.; Peterson, P.; Weckesser, W.; Bright, J.; van der Walt, S. J.; Brett, M.; Wilson, J.; Millman, K. J.; Mayorov, N.; Nelson, A. R. J.; Jones, E.; Kern, R.; Larson, E.; Carey, C. J.; Polat, I.; Feng, Y.; Moore, E. W.; VanderPlas, J.; Laxalde, D.; Perktold, J.; Cimrman, R.; Henriksen, I.; Quintero, E. A.; Harris, C. R.; Archibald, A. M.; Ribeiro, A. H.; Pedregosa, F.; van Mulbregt, P. SciPy 1.0: Fundamental Algorithms for Scientific Computing in Python. Nat. Methods 2020, 17 (3), 261-272.

(48) Sheu, S.-Y.; Yang, D.-Y.; Selzle, H. L.; Schlag, E. W. Energetics of Hydrogen Bonds in Peptides. Proc. Natl. Acad. Sci. 2003, 100 (22), 12683-12687.

(49) Kim, D.; Wagner, N.; Wooding, K.; Clemmer, D. E.; Russell, D. H Ions from Solution to the Gas Phase: A Molecular Dynamics Simulation of the Structural Evolution of Substance P during Desolvation of Charged Nanodroplets Generated by Electrospray Ionization. J. Am. Chem. Soc. 2017, 139 (8), 2981-2988.

(50) Shi, L.; Holliday, A. E.; Bohrer, B. C.; Kim, D.; Servage, K. A.; Russell, D. H.; Clemmer, D. E. "Wet" Versus "Dry" Folding of Polyproline. J. Am. Soc. Mass Spectrom. 2016, 1037-1047.
(51) Jarrold, M. F. Helices and Sheets in Vacuo. Phys. Chem. Chem. Phys. 2007, 9, 1659-1671.

(52) Trimpin, S.; Clemmer, D. E. Ion Mobility Spectrometry/Mass Spectrometry Snapshots for Assessing the Molecular Compositions of Complex Polymeric Systems. Anal. Chem. 2008, 80 (23), 9073-9083.

(53) De Winter, J.; Lemaur, V.; Ballivian, R.; Chirot, F.; Coulembier, O.; Antoine, R.; Lemoine, J.; Cornil, J.; Dubois, P.; Dugourd, P.; Gerbaux, P. Size Dependence of the Folding of Multiply Charged Sodium Cationized Polylactides Revealed by Ion Mobility Mass Spectrometry and Molecular Modelling. Chem. - A Eur. J. 2011, 17 (35), 9738-9745.

(54) Pierson, N. A.; Valentine, S. J.; Clemmer, D. E. Evidence for a QuasiEquilibrium Distribution of States for Bradykinin $[\mathrm{M}+3 \mathrm{H}] 3+$ Ions in the Gas Phase. J. Phys. Chem. B 2010, 114 (23), 7777-7783.

(55) Morsa, D.; Gabelica, V.; De Pauw, E. Effective Temperature of Ions in Traveling Wave Ion Mobility Spectrometry. Anal. Chem. 2011, 83 (14), 5775-5782.

(56) Morsa, D.; Hanozin, E.; Eppe, G.; Quinton, L.; Gabelica, V.; Pauw, E. De. Effective Temperature and Structural Rearrangement in Trapped Ion Mobility Spectrometry. Anal. Chem. 2020, 92 (6), 4573-4582.

(57) Naylor, C. N.; Ridgeway, M. E.; Park, M. A.; Clowers, B. H. Evaluation of Trapped Ion Mobility Spectrometry Source Conditions Using Benzylammonium Thermometer Ions. J. Am. Soc. Mass Spectrom. 2020, 31 (7), 1593-1602.

(58) Bleiholder, C.; Liu, F. C.; Chai, M. Comment on Effective Temperature and Structural Rearrangement in Trapped Ion Mobility Spectrometry: TIMS Enables Native Mass Spectrometry Applications. Anal. Chem. 2020, acs.analchem.0c02052. (59) Pauw, E. De; Morsa, D.; Hanozin, E. Response to Comment on Effective Temperature and Structural Rearrangement in Trapped Ion Mobility Spectrometry. Anal. Chem. 2020, 92, 16334-16337. 


\section{Supporting Information}

\section{Contents:}

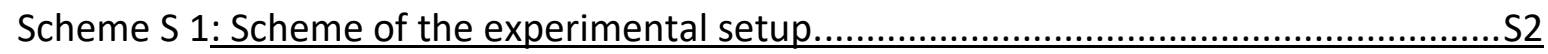

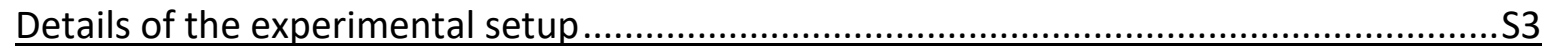



Two-state model and population equations ............................................................ 5

Figure S 1: Arrival time distributions for doubly-protonated GluFib2+ ions with and without

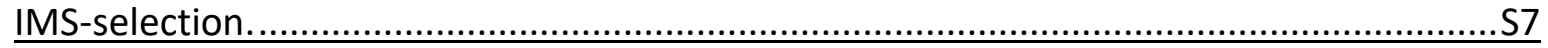

Figure S 2: Arrival time distributions for doubly-protonated GluFib2+ ions with Gaussian fits.

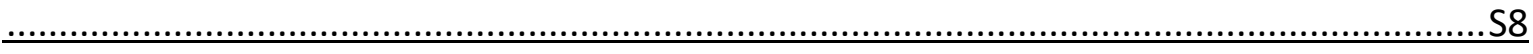

Figure S 3: Evolution of the relative proportion of population $A$ as a function of trapping time

for different trapping temperatures. ................................................................ 9

Figure S 4: Gyration radii distributions from structures sampled along the REMD production

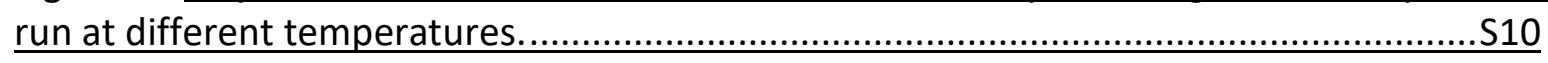

Figure S 5: Comparison of the lowest laying isomers for clusters $A, B 1$, and $B 2 \ldots \ldots \ldots \ldots . . . . .511$

Figure S 6: Free energy surfaces generated by WHAM. .............................................. 12

Figure S 7: Representation of the lowest energy structure for cluster A and cluster B2. S13

Figure S 8: Distribution of selected structural observables among inherent structures at

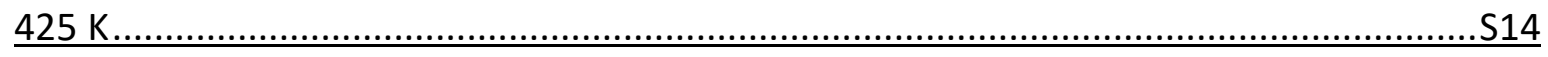




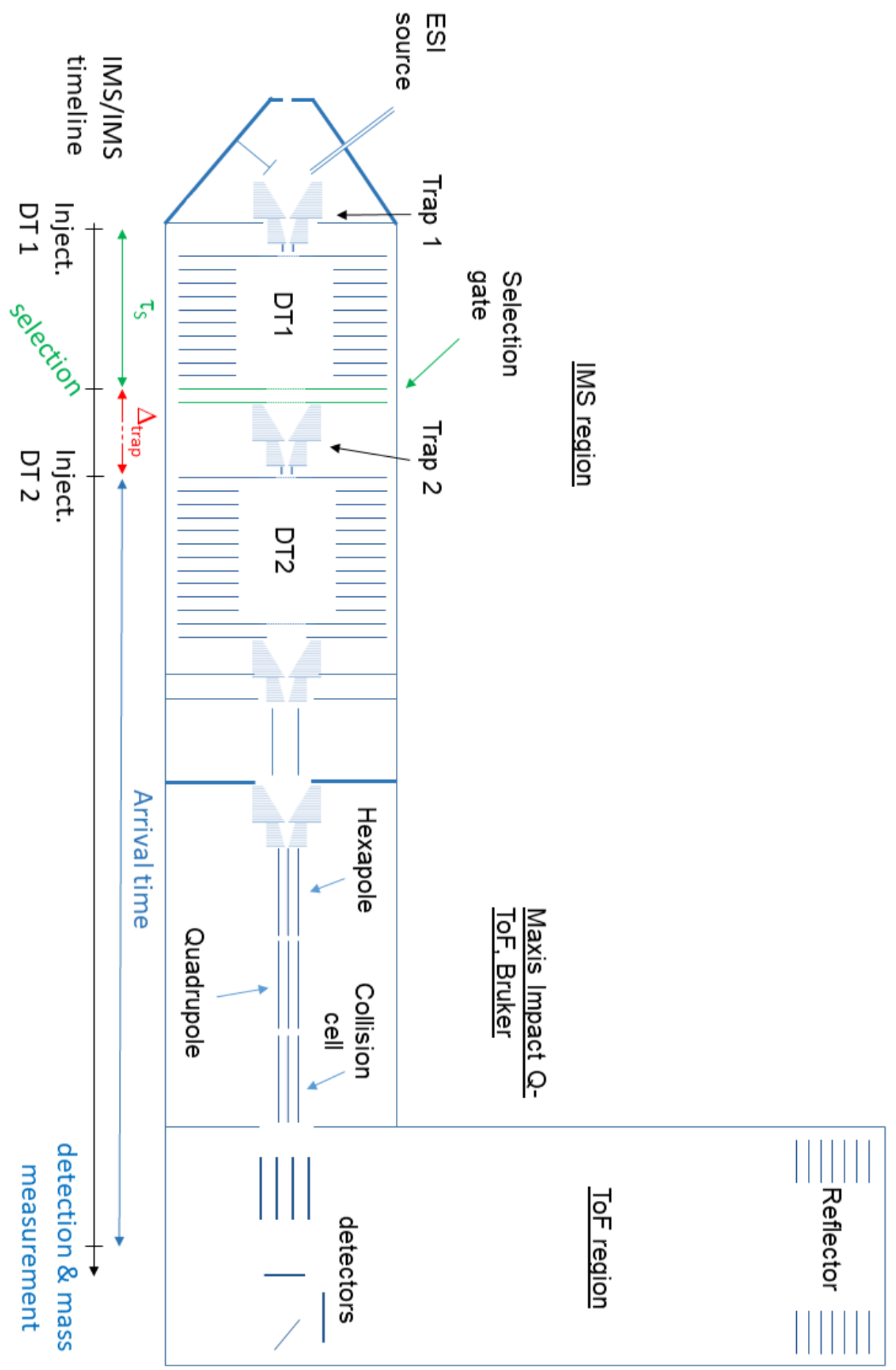

Scheme S 1 : Scheme of the experimental setup with a timeline describing the operation in the IMS/IMS mode. 


\section{Details on the experimental setup}

Our IMS/IMS instrument was described in details in ref. 1. It is equipped with an electrospray ion source, which was operated in the positive ion mode. For the present experiments the drift region of the instrument was filled with 3.7 Torr helium maintained at $11^{\circ} \mathrm{C}$ through the circulation of silicon oil (sil180, Thermo Fisher) within the walls of the vacuum chamber. The drift region is separated into two identical $79 \mathrm{~cm}$ long sections (DT1 and DT2 in Scheme S1). A constant electric field is applied across each section for IMS separation. At each end of DT1 and DT2, a dual ion funnel assembly allows for ion focusing and trapping.

Drift time measurements begin by injecting short ( $200 \mu \mathrm{s})$ ion bunches out of one of the trapping regions of the ion funnels, denoted Trap 1 and Trap 2 in Scheme S1. For detection, we use the time-of-flight (TOF) mass spectrometer from a Maxis Impact (Bruker, Bremen, Germany) coupled downstream the drift region. The TOF extraction is synchronized with the injection so that a mass spectrum can be recorded every $100 \mu \mathrm{s}$. This defines the sampling time for arrival time distributions (ATDs), which are extracted by integrating mass ranges of interest across the time-referenced mass spectra.

Two distinct operation modes were used for the present work. For single IMS measurements, Trap 1 is continuously opened and ion bunches are injected from Trap 2, into DT2. To determine IMS cross sections (CCS), we record ATDs at, typically 6, different drift voltages from 200 to $550 \mathrm{~V}$, across DT2. The CCS value is finally obtained, without any calibration, from the variation rate of the arrival time as a function of the inverse drift field, based on the fundamental mobility equation, and assuming weak field conditions are fullfilled. ${ }^{2,3}$

For IMS-IMS measurement (see timeline in Scheme S1), ions are injected from Trap 1. They are selected as a function of their drift time across DT 1 by opening the ion gate within the desired time window $\left(\left[\tau_{S} ; \tau_{S}+\delta_{S}\right]\right)$. For the present experiments, $\delta_{S}$ was $500 \mu \mathrm{s}$, which is about the with expected for a diffusion-limited ion cloud at the end of the first drift tube. Selected ions are then stored in Trap 2 for a controlled duration $\left(\Delta_{\text {trap }}\right)$ before injection in DT2. In this mode, the arrival time refer to the drift across DT 2 only, and are thus the same as those measured in the single IMS mode. An activation zone is placed immediately after the selection gate. It consists in two high-transmission nickel grids separated by $5 \mathrm{~mm}$. A voltage can be applied between these grids to perform collisional activation of IMS-selected ions.

\section{Thermalization in Trap 2}

Trap 2 is composed of 14 aluminium electrodes $(0.5 \mathrm{~mm}$ thick, with a $3 \mathrm{~mm}$ diameter circular hole at their center, and spaced by $0.8 \mathrm{~mm}$ ). It is housed in a cylindrical peek chamber whose only apertures ( $1 \mathrm{~mm}$ diameter each) correspond to that of the two DC-only electrodes upstream and downstream the trap (the latter serves as an ion gate for injection in DT 2). A $25 \mathrm{~cm}$ long, $1 \mathrm{~mm}$ thick, ceramic-insulated resistive heater (Thermocoax) was co-axially wired around the electrodes. The heating power was adjusted by tuning the current flowing through the $3 \Omega$ heating element (typically 1-2.5 A). The temperature was measured by a thermocouple in thermal contact with one of the electrodes. The uncertainty on the temperature was estimated to be $\pm 2 \mathrm{~K}$ from series of measurements at constant heating power. Since the thermalization of the buffer gas by collisions on the electrodes is expected to be much faster than that of the electrodes themselves, we assume that the gas inside the trap 
is in thermal equilibrium with the electrodes, and that the temperature measured on the electrode reflects the temperature of the gas.

We assume that the buffer gas inside the trap acts as an efficient heat bath for the ions. Although no accurate model is available to estimate the efficiency of heat transfer in ion/neutral collisions, the heat bath hypothesis proved to be valid for peptide ions trapped in similar conditions. ${ }^{4}$ Importantly, we did not notice any observable effect (isomerization or fragmentation) of potential heating induced by the radiofrequency (RF) confining voltages in our setup. In a previous study, Bush and co workers estimated the increase in the effective temperature of ions in a RF-confined drift tube (i.e. in similar experimental conditions) to be less than $2 \mathrm{~K},{ }^{5}$ which lies within our experimental error bars.

\section{Replica exchange molecular dynamics}

Replica-exchange molecular dynamics simulations (REMD) ${ }^{6}$ were performed to explore the conformational landscape of the investigated peptide, and produce ensembles of structures and observables comparable to our experimental data. ${ }^{7}$ We used a custom python implementation of REMD based on the python plugin of the OpenMM library. ${ }^{8}$ Simulations were performed in vacuo with the AMOEBA polarizable force field. ${ }^{9}$ Thermalization was achieved through the Langevin integrator implemented in OpenMM, using a $1 \mathrm{ps}^{-1}$ damping constant and a time step of $1 \mathrm{fs}$. For REMD, 16 trajectories were propagated in parallel, at different temperatures ranging from $300 \mathrm{~K}$ to $800 \mathrm{~K}$, distributed following a geometric progression. An exchange between random adjacent replicas was attempted every 100 step, accepted based on a Metropolis criterion.

The trajectories were initially seeded with a totally unfolded structure of doubly-protonated GluFib, generated using the Gabedit software. ${ }^{10}$ Based on basicity considerations, and to reproduce the observed charge state, we assumed the $\mathrm{N}$-terminal amine and the final Arginine were protonated, and then positively charged. All carboxyl groups on the C-terminal moiety and on the side chains of the acidic residues were considered protonated and neutral, respectively. After 20 ps equilibration, structures were periodically sampled during a 1 ns MD run. A local optimization was finally performed on the sample structures in order to identify the global minimum. The procedure was repeated by seeding the new trajectories with the putative global minimum. No change was observed in the gyration radii distributions of the sampled structures, nor in the global minimum after 3 such cycles. A longer production cycle was finally run for $6 \mathrm{~ns}$, yielding 30000 sample structures from each replica.

Two-states model and population equations

To model the time evolution of the two populations, A and B, observed in our experiment, we considered a two-state model in which isomerization is possible in both directions with different rate constants. If $I_{A}(t)$ and $I_{B}(t)$ correspond to the population in each state at time $t$, rate equations for their evolution can be written as:

$$
\left\{\begin{array}{l}
\frac{\mathrm{d} I_{A}}{\mathrm{~d} t}=-k_{A B} I_{A}+k_{B A} I_{B} \\
\frac{\mathrm{d} I_{B}}{\mathrm{~d} t}=k_{A B} I_{A}-k_{B A} I_{B}
\end{array}\right.
$$


where $k_{A B}$ and $k_{B A}$ respectively correspond to the isomerization rate constant from $\mathrm{A}$ to $\mathrm{B}$, and from $B$ to $A$, at temperature $T$.

In our experiment, IMS selection allows to define the populations at $\mathrm{t}=0$. For example, selecting population B defines $I_{A}(t=0)=0$ and $I_{B}(t=0)=1$. In this case the solutions of equation (S1) can be written as:

$$
\left\{\begin{array}{l}
I_{A}(t)=C_{A B}\left(1-e^{-k t}\right) \\
I_{B}(t)=C_{A B} e^{-k t}+C_{B A}
\end{array}\right.
$$

where the constants depend only on the rates $k_{A B}$ and $k_{B A}: k=k_{A B}+k_{B A}, C_{A B}=k_{A B} / k$, and $C_{B A}=k_{B A} / k$.

The time evolution of each of the populations can thus be fitted using equation (S2), with $k_{A B}$ and $k_{B A}$ as free parameters. Arrhenius activation energies $\mathrm{E}_{A B}^{*}$ and $\mathrm{E}_{B A}^{*}$ can finally be extracted from the evolution of these rate constants as a function of temperature. Finally, for long enough timescales $(t \gg 1 / k)$, the populations reach a steady state, which we considered as an equilibrium. Consequently, the quantity $K_{A B}=I_{B}(\infty) / I_{A}(\infty)=k_{A B} / k_{B A}$ correspond to an equilibrium constant, whose evolution as a function of temperature is ruled by:

$$
K_{A B}(T)=e^{-\Delta \mathrm{G}_{\mathrm{AB}} / R T}
$$

where $R$ is the ideal gas constant, and $\Delta \mathrm{G}_{\mathrm{AB}}=\Delta \mathrm{H}_{\mathrm{AB}}-T \Delta \mathrm{S}_{\mathrm{AB}}$ is the Gibbs free energy for the isomerization reaction. Equation (S3) finally allows to estimate $\Delta \mathrm{H}_{\mathrm{AB}}$ and $\Delta \mathrm{S}_{\mathrm{AB}}$, the enthalpy and entropy of isomerization, from our temperature-dependent kinetics measurements. 


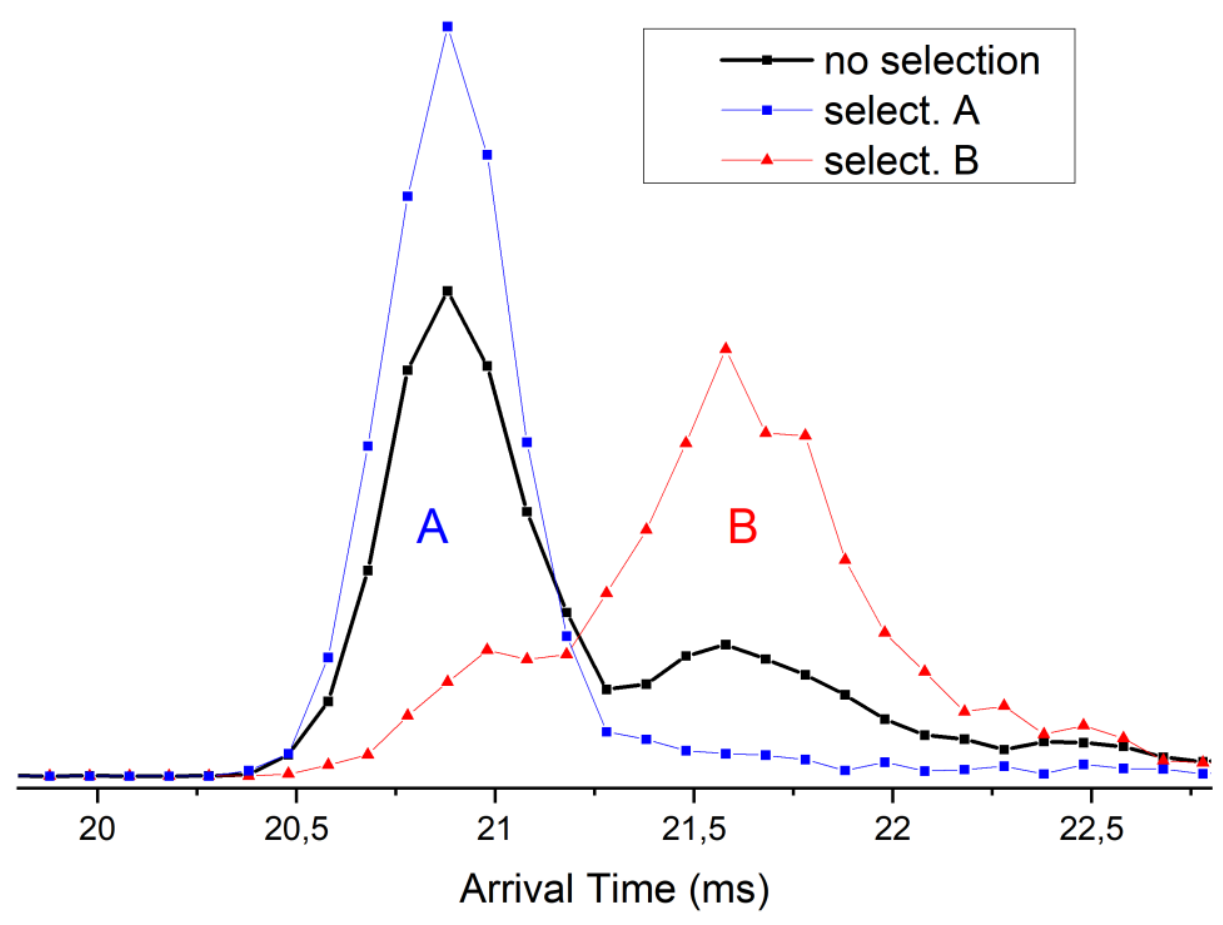

Figure S 1: Arrival time distributions for doubly-protonated $\mathrm{GluFib}^{2+}$ ions recorded without IMS selection (black), and after selection of population A (blue), or B (red). Drift conditions: voltage $450 \mathrm{~V}$, drift gas: 3.7 Torr Helium at $284 \mathrm{~K}$. 


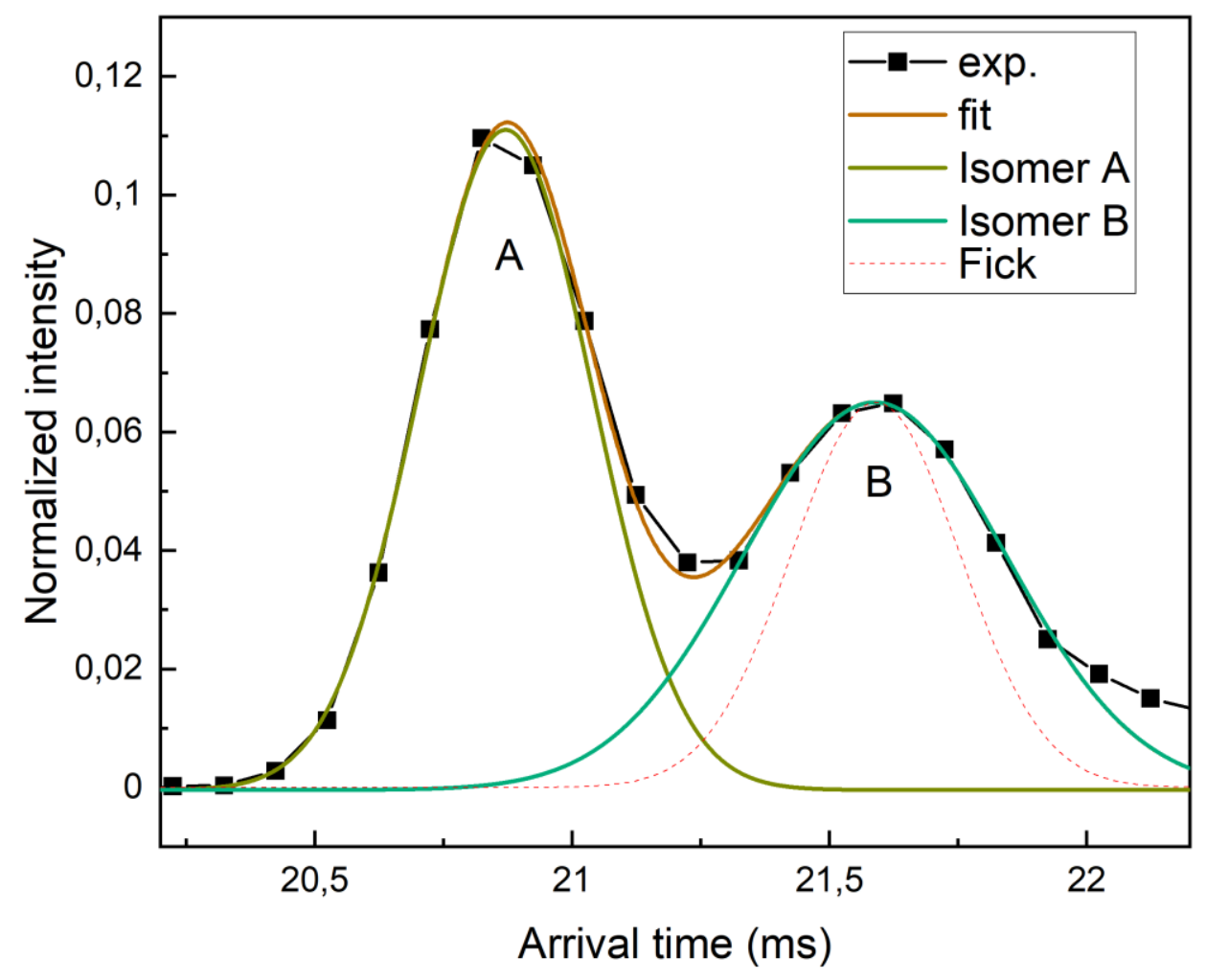

Figure S 2 : Arrival time distributions for doubly-protonated $\mathrm{GluFib}^{2+}$ ions. Drift conditions: voltage $450 \mathrm{~V}$, drift gas: 3.7 Torr Helium at $284 \mathrm{~K}$. A and B denote the two considered populations. The solid line represent the Gaussian functions used to fit each population (gold, and blue), and the sum of the two contributions (orange). The red dotted line correspond to the shape expected for a diffusion-limited peak. 

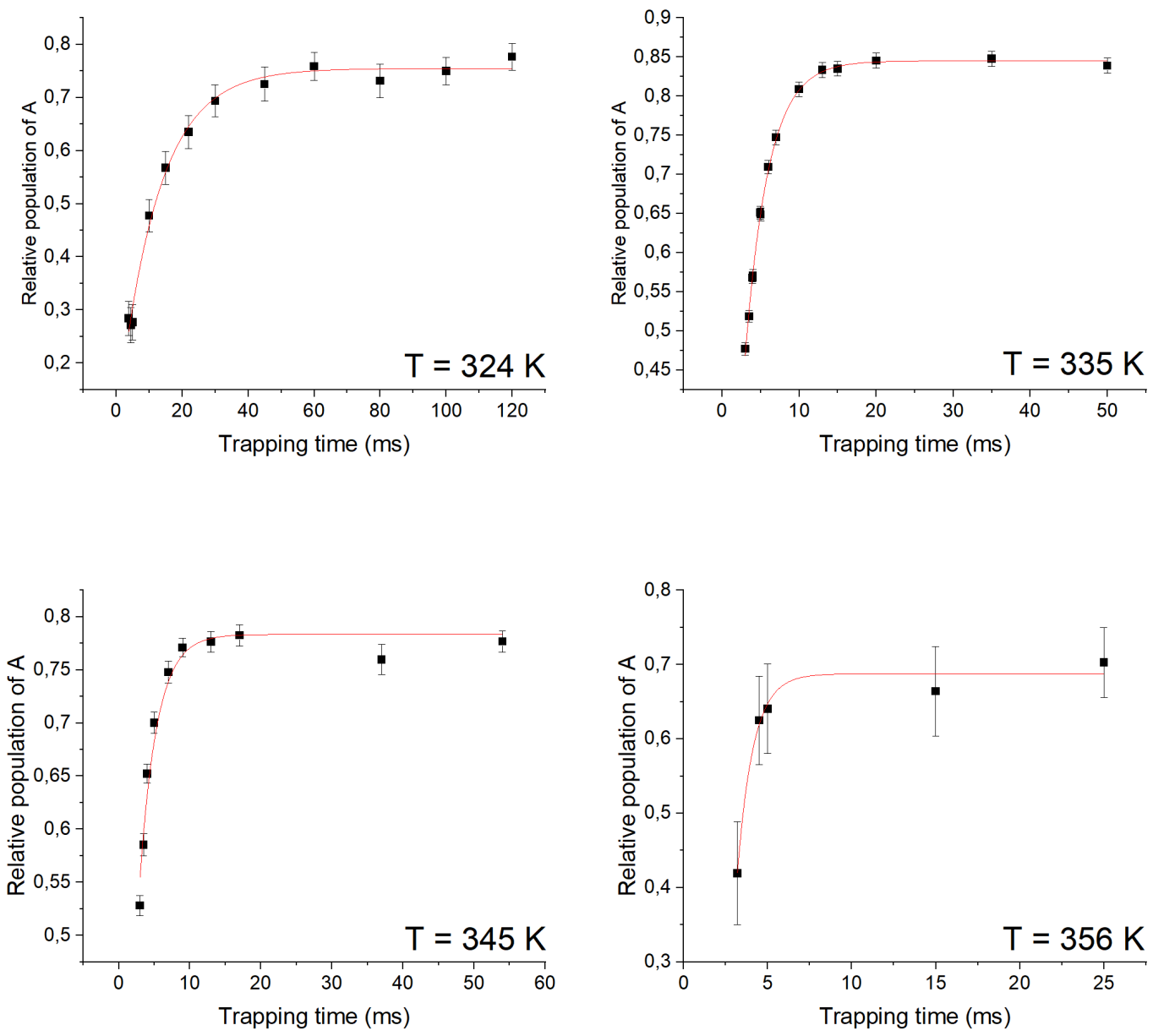

Figure $S 3$ : Evolution of the relative proportion of population $A$ as a function of trapping time for different trapping temperatures. The solid line correspond to a fit of the data using equation (S2). 

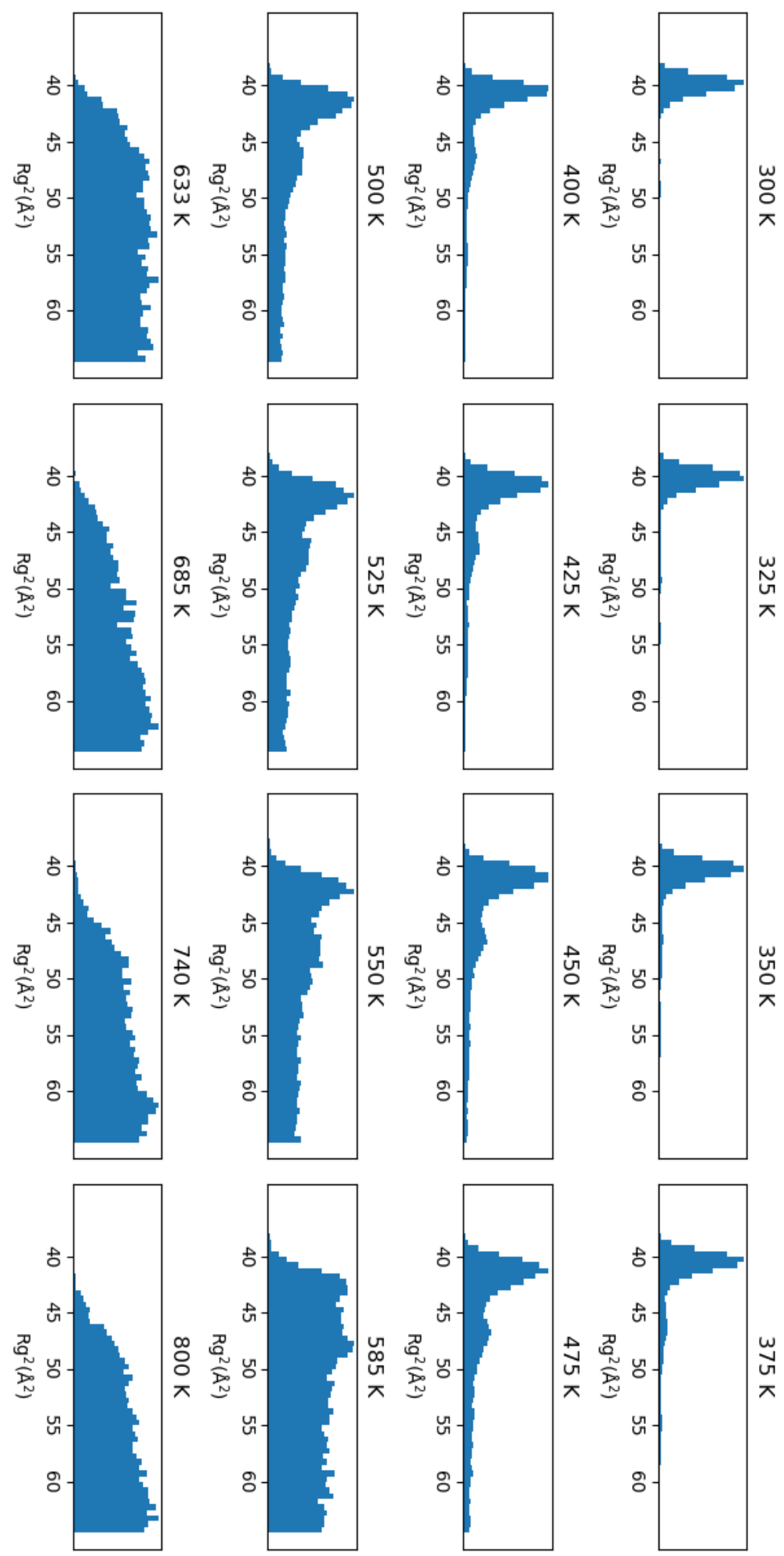

Figure S 4: Gyration radii distributions from 30000 structures sampled along the different replica of the 6 ns REMD production run. 


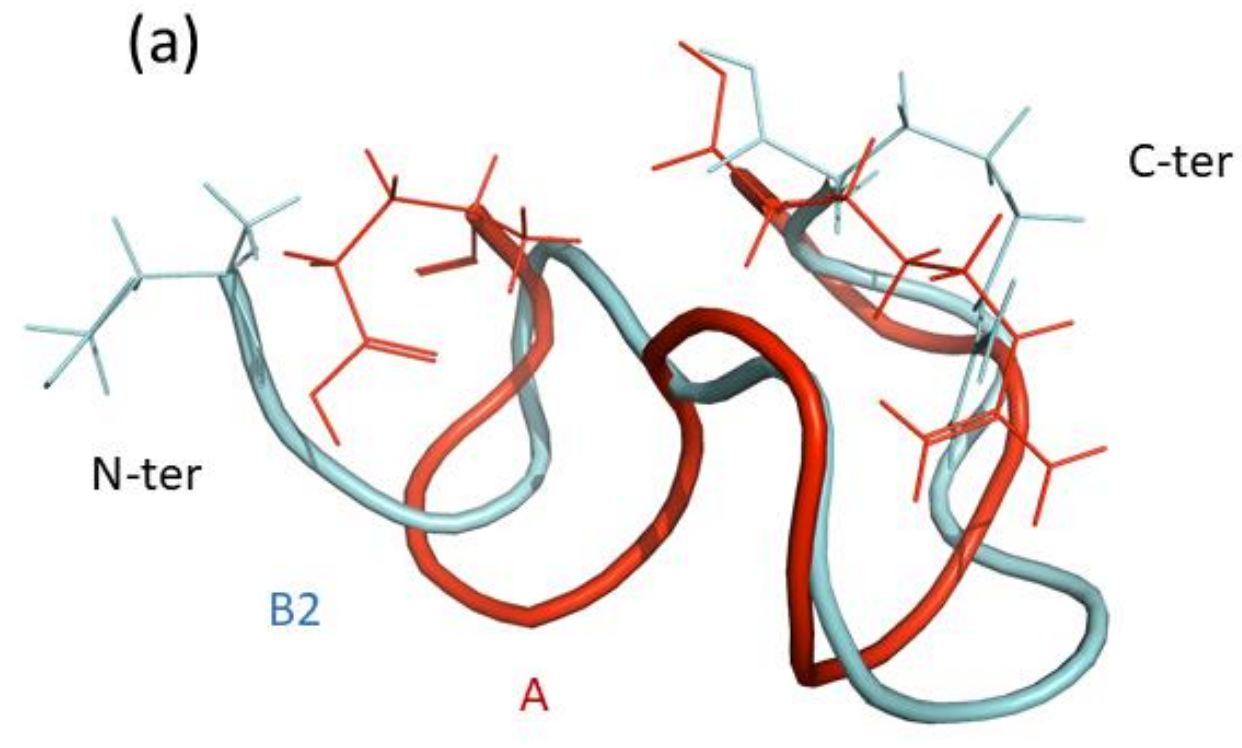

(b)

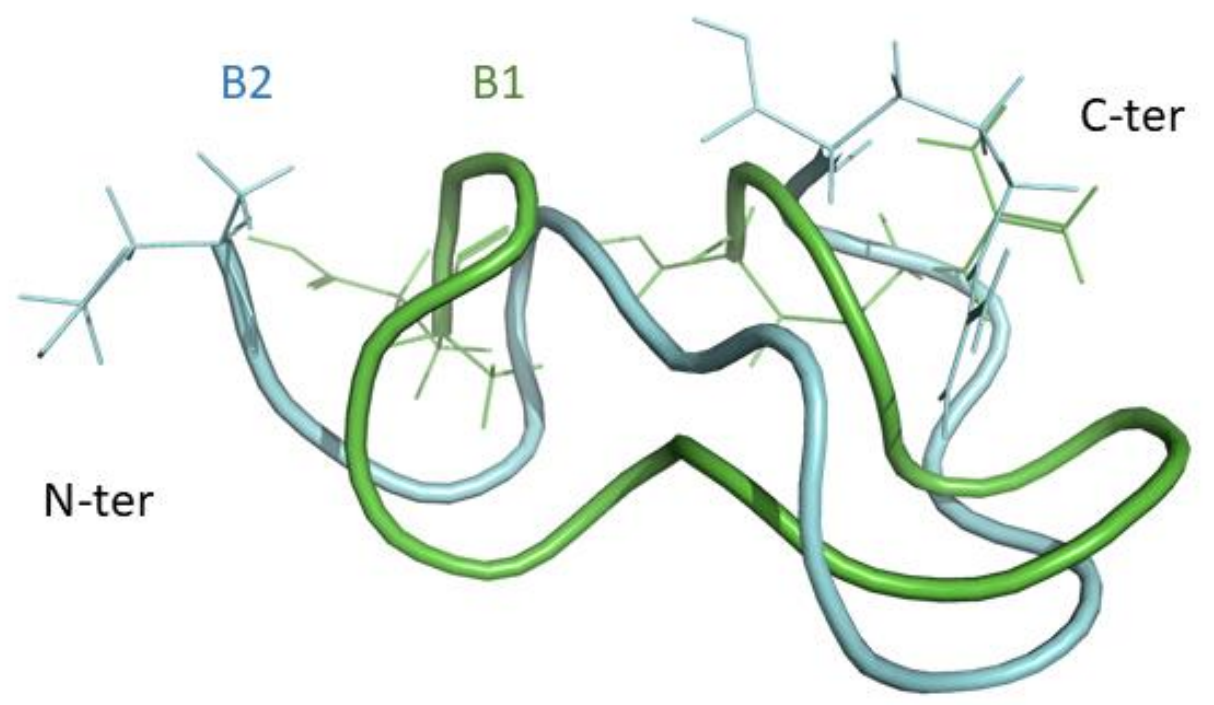

Figure S 5: Sketch (backbone and side chains of the terminal amino acids) of the lowestlaying structures from clusters $A$ (red), B1 (green), and B2 (cyan), denoted $A_{0}, B_{1}$, and $B 2_{0}$, respectively. (a) Superimposition of $A_{0}$ and $B 2_{0}$ emphasizes the relative structural similarity on the C-terminus side of the peptide chain, as well as the unfolding of $B 2_{0}$ on the $\mathrm{N}$ terminal side. (b) Superimposition of $\mathrm{B} 1_{0}$ and $\mathrm{B} 2_{0}$ shows that $\mathrm{B} 1_{0}$ is globally more compact, but with a completely different arrangement of its backbone. 

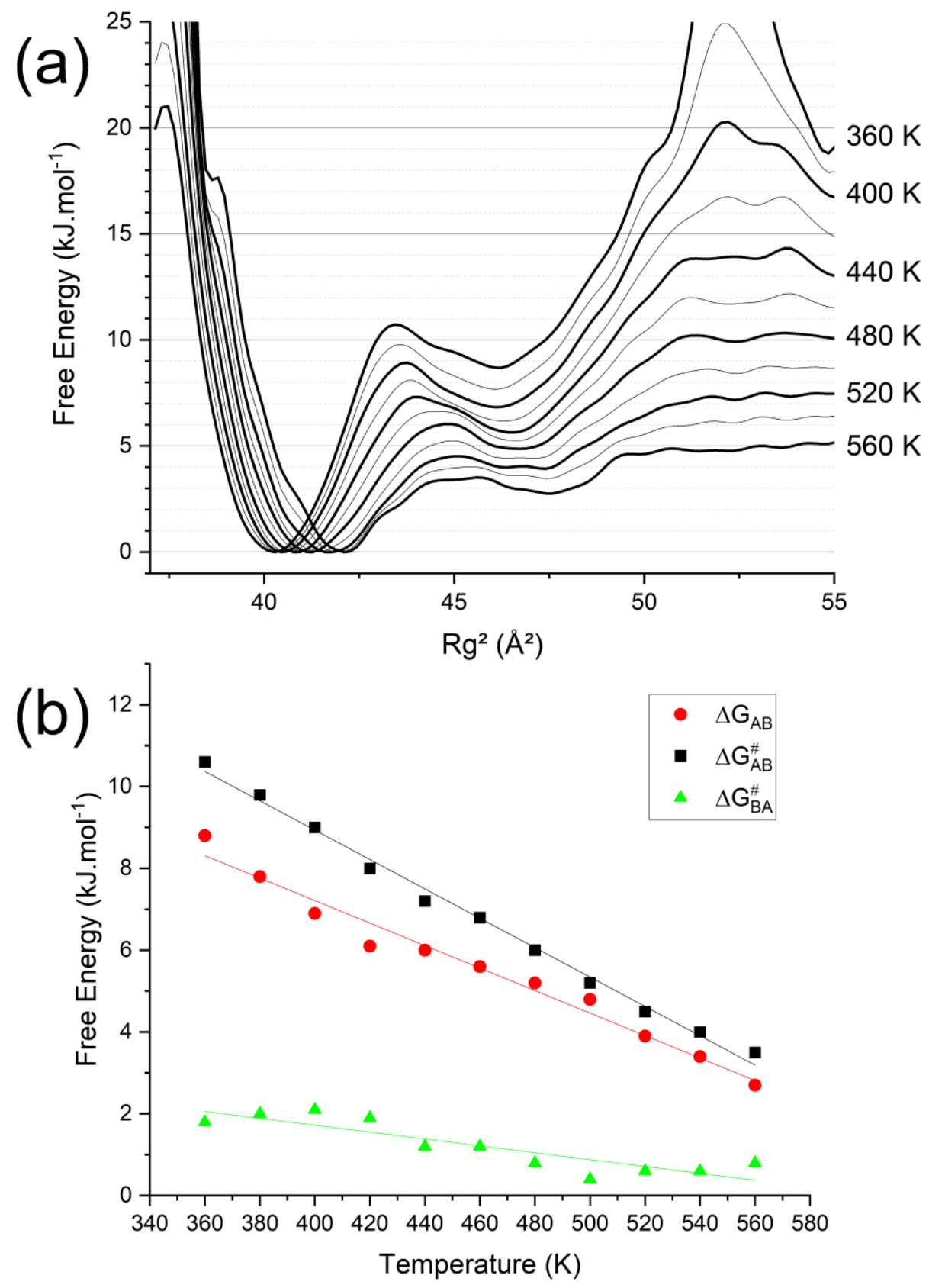

Figure S 6: (a) Free energy as a function of the radius of gyration computed using the WHAM procedure for evenly spaced temperatures on the range $360 \mathrm{~K}-560 \mathrm{~K}$. The surfaced were smoothed using a Savitszky-Golay adjacent averaging procedure with a 3 pts window. (b) Free energy differences between the bottom of the two wells (red circles), and between the bottom of each well and the top of the intermediate barrier (black squares, and green triangles), as a function of temperature. The lines result from a linear fit. 

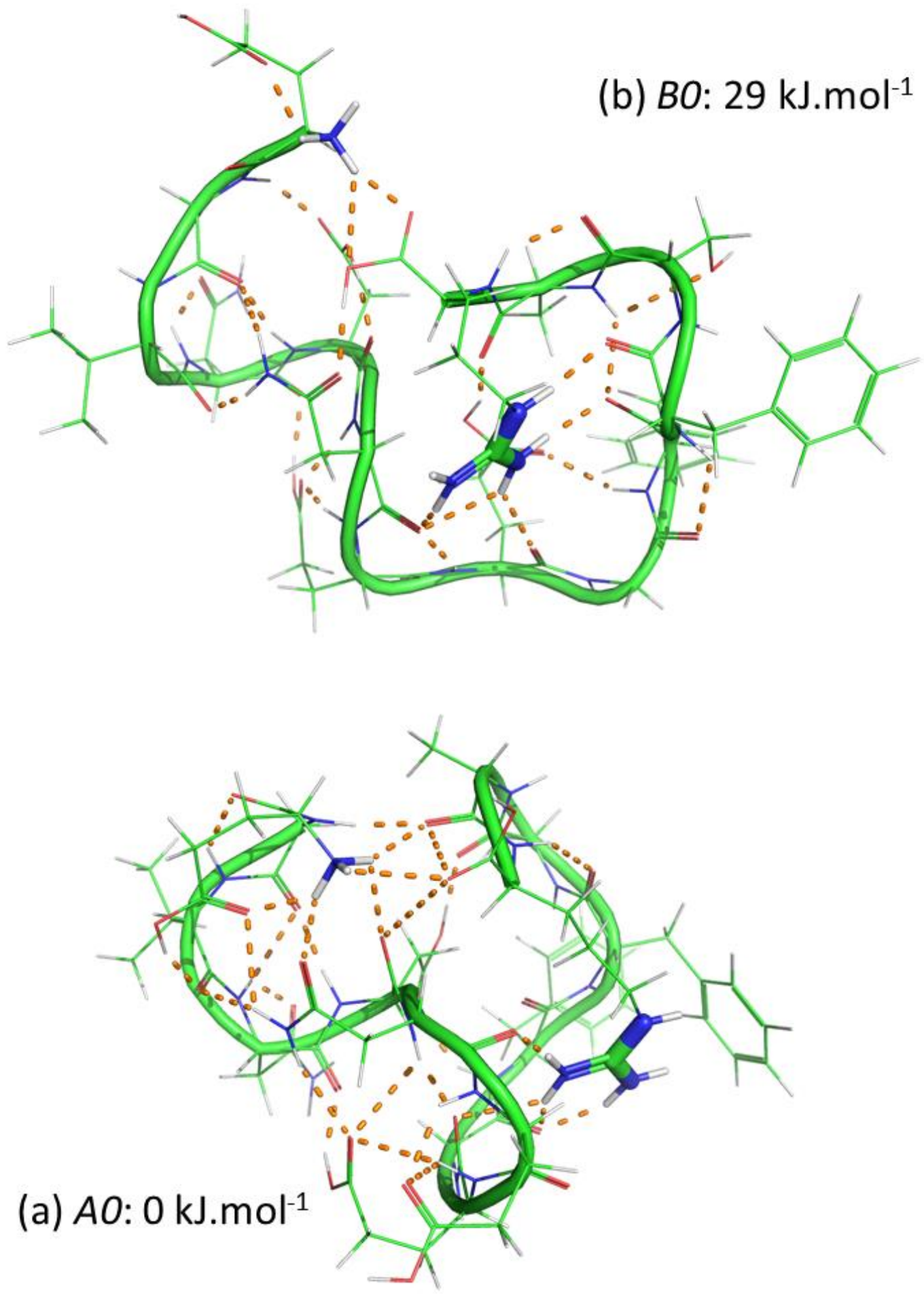

Figure S 7 : representation of the lowest energy structure for cluster A (a), and cluster B2 (b) with their energy relative to the global minimum. Contacts between polar moieties are highlighted as orange dotted lines. The two protonation sites on the $\mathrm{N}$-terminal amine and the Arg14 side chain are highlighted with bold sticks. 
(a)

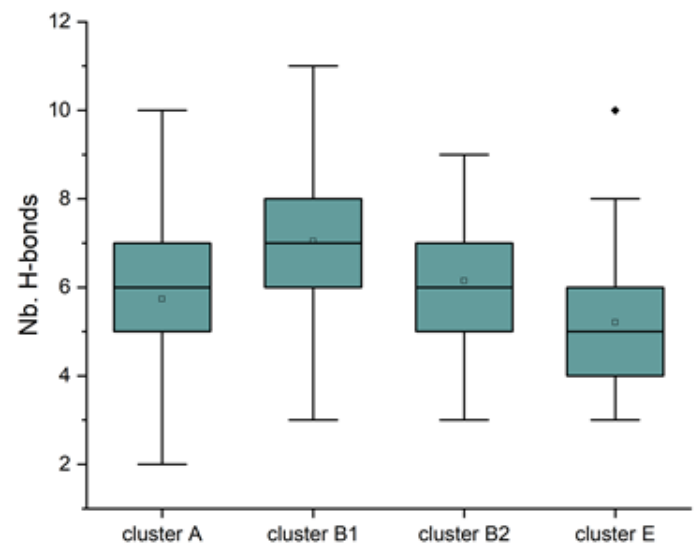

(b)

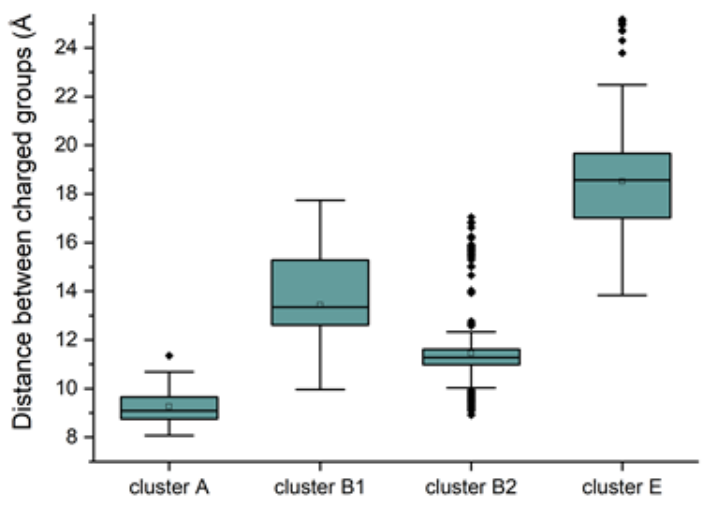

(c)

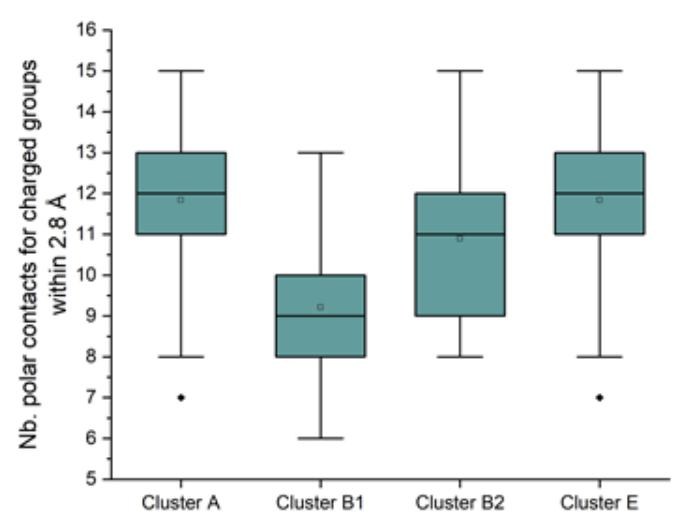

Figure S 8: Box plots for the distributions of selected structural observables among inherent structures determined for the REMD run at $425 \mathrm{~K}$, grouped in clusters. (a) number of $\mathrm{H}$ bonds among inherent structures determined for the REMD run at $425 \mathrm{~K}$, grouped in clusters; (b) distance between the nitrogen atom of the N-terminal amino group and the $\mathrm{CZ}$ atom in the side chain of Arg14; (c) number of oxygen atoms within $2.8 \AA$ of an hydrogen belonging to one of the charged moieties. 


\section{References}

(1) Simon, A.-L.; Chirot, F.; Choi, C. M.; Clavier, C.; Barbaire, M.; Maurelli, J.; Dagany, X.; MacAleese, L.; Dugourd, P. Tandem Ion Mobility Spectrometry Coupled to Laser Excitation. Rev. Sci. Instrum. 2015, 86 (9), 094101. https://doi.org/10.1063/1.4930604.

(2) Revercomb, H. E.; Mason, E. A. Theory of Plasma Chromatography, Gazeous Electrophoresis - Review. Anal. Chem. 1975, 47 (7), 970-983. https://doi.org/10.1021/ac60357a043.

(3) Siems, W. F.; Viehland, L. A.; Hill, H. H. Correcting the Fundamental Ion Mobility Equation for Field Effects. Analyst 2016, 141 (23), 6396-6407. https://doi.org/10.1039/C6AN01353H.

(4) Butcher, D. J.; Asano, K. G.; Goeringer, D. E.; McLuckey, S. A. Thermal Dissociation of Gaseous Bradykinin lons. J. Phys. Chem. A 1999, 103 (43), 8664-8671. https://doi.org/10.1021/jp9924979.

(5) Allen, S. J.; Bush, M. F. Radio-Frequency (Rf) Confinement in Ion Mobility Spectrometry: Apparent Mobilities and Effective Temperatures. J. Am. Soc. Mass Spectrom. 2016, 27 (12), 2054-2063. https://doi.org/10.1007/s13361-016-1479-9.

(6) Sugita, Y.; Okamoto, Y. Replica-Exchange Molecular Dynamics Method for Protein Folding. Chem. Phys. Lett. 1999, 314 (1-2), 141-151. https://doi.org/doi: 10.1016/S0009-2614(99)01123-9.

(7) Chirot, F.; Calvo, F.; Albrieux, F.; Lemoine, J.; Tsybin, Y. O.; Dugourd, P. Statistical Analysis of Ion Mobility Spectrometry. I. Unbiased and Guided Replica-Exchange Molecular Dynamics. J. Am. Soc. Mass Spectrom. 2012, 23 (2), 386-396. https://doi.org/10.1007/s13361-011-0281-y.

(8) Eastman, P.; Swails, J.; Chodera, J. D.; McGibbon, R. T.; Zhao, Y.; Beauchamp, K. A.; Wang, L.-P.; Simmonett, A. C.; Harrigan, M. P.; Stern, C. D.; et al. OpenMM 7: Rapid Development of High Performance Algorithms for Molecular Dynamics. PLOS Comput. Biol. 2017, 13 (7), e1005659. https://doi.org/10.1371/journal.pcbi.1005659.

(9) Shi, Y.; Xia, Z.; Zhang, J.; Best, R.; Wu, C.; Ponder, J. W.; Ren, P. Polarizable Atomic Multipole-Based AMOEBA Force Field for Proteins. J. Chem. Theory Comput. 2013, 9 (9), 4046-4063. https://doi.org/10.1021/ct4003702.

(10) Allouche, A. R. Gabedit-A Graphical User Interface for Computational Chemistry Softwares. J. Comput. Chem. 2011, 32, 174-182. https://doi.org/10.1002/jcc.21600. 